\title{
Lits fluidisés pour l'industrie chimique. Extrapolation et amélioration des catalyseurs Première partie : Études et modèles - Enseignements issus des pilotes
}

\author{
R. Botton \\ 32, rue Garibaldi, 69006 Lyon - France
}

\begin{abstract}
Résumé - Les unités de production en lits fluidisés catalytiques sont apparues vers 1942 dans l'industrie pétrolière et vers 1960 dans l'industrie chimique. On se limitera ici au problème de l'extrapolation des lits fluidisés catalytiques pour l'industrie chimique, qui exigent de très hautes performances (> $99 \%$ de conversion). Leur mise au point a, dans le passé, nécessité l'exploitation sur des sites industriels de coûteux pilotes de $0,5 \mathrm{~m}$ de diamètre et de plus de $10 \mathrm{~m}$ de hauteur. Nous montrerons que ces pilotes peuvent être évités et que le passage direct du laboratoire à l'échelle industrielle est réalisable. Cette possibilité offre en plus une méthode simple pour améliorer les catalyseurs des unités industrielles. Elle ouvre aussi cette technique, très appréciée en production, aux produits de petits tonnages. La présentation de cet article sera faite en trois parties :

- La première présentée ci-après expose les problèmes majeurs posés par l'extrapolation, puis résume les études effectuées. Les travaux d'extrapolation relatifs à deux procédés effectués avec des pilotes sont ensuite présentés, à titre d'exemples. De ces travaux, sont déduites les performances que l'on peut espérer obtenir avec un réacteur catalytique à lit fluidisé, ainsi que les règles de tendances à suivre pour $\mathrm{y}$ parvenir.

- La deuxième partie intitulée Stratégie n'utilisant que des expériences de laboratoire propose une stratégie expérimentale permettant d'obtenir en laboratoire les informations nécessaires pour passer directement à l'échelle industrielle avec des expériences suggérées en partie par les résultats exposés dans le premier article. Les relations expérimentales établies lors de ces études montrent que les propriétés d'un lit fluidisé ne dépendent (mis à part quelquefois le diamètre du réacteur) que d'un paramètre appelé « vitesse minimum de fluidisation de comportement ».

- La troisième partie est intitulée Études théoriques, réalités expérimentales, suggestions. Les bulles des lits fluidisés ont fait l'objet de très nombreux travaux, dont les résultats sont très souvent explicités sous la forme de modèles mécanistiques à un paramètre qui est le diamètre des bulles. Pour confronter ces modèles à l'expérience, une relation est établie entre le diamètre des bulles et la vitesse minimum de fluidisation de comportement. Des suggestions sont alors faites pour améliorer les modèles, et l'on propose des conclusions générales sur les lits fluidisés.
\end{abstract}

Mots-clés : lits fluidisés catalytiques, extrapolation, amélioration des catalyseurs, modèles, reacteurs.

Abstract - Fluidized Beds in Chemical Industry. Scale up and Catalysts Improvement - The firsts catalytic fluidized beds appear near 1942 in petroleum industry and near 1960 in chemical industry. We only consider very high performances chemical fluidized bed reactors (> 99\%). 
In the past, they were developed through the use of very expensive pilot plants of about $0.5 \mathrm{~m}$ diameter and $10 \mathrm{~m}$ high. We will demonstrate that direct scale up from laboratory data is possible. This possibility gives also a simple method to improve catalysts used into operating units and opens fluidized bed technique to products that need only low production. Presentation is made with three articles:

- In the first, "Studies, Models, Learning from Pilot Plants": after a description of the major scale-up problems, studies to solve then are summarized. Then scale-up works of two processes with the use of about 0.5 m diameter pilot plant are given. From the results it is deduced the possible performances of a catalytic fluidized bed and how to operate to obtain then.

- In the second "Scale up with Only Laboratory Data", it is experimentally demonstrated that the information's scale-up can be optained in a laboratory. A strategy to obtain them is suggested. An another result of theses experimental studies is that all physical properties of catalytic fluidized bed depends of only one parameter. It is called "comportment incipient fluidization velocity”.

- In the third "Theoretical Studies, Experimental Reality, Suggestions" results of theoretical studies about bubbles of the fluidized beds are summarized. Results are often given through one parameter mechanistic models. This parameter is the bubble diameter. To confront theses mechanistic models to experiences a relation is established between the bubble diameter and the comportment incipient velocity fluidization. Suggestions to improve bubble models are suggested and general conclusions about fluidized beds are given.

Keywords: catalytic fluidized bed, scale-up, improvement of catalysts, models, reactors.

\section{NOMENCLATURE}

Le système d'unités de base utilisé est le système $\mathrm{SI}(\mathrm{kg}, \mathrm{m}, \mathrm{s})$.

À noter : vitesse superficielle $=$ débit par unité de section de l'appareil.

$C_{o}, C_{S}$

concentrations du réactif à l'entrée et à la sortie du lit $\left(\mathrm{mol} / \mathrm{m}^{3}\right)$

$d p_{10} \quad$ ouverture du tamis qui laisse passer $10 \%$ (en poids) d'une poudre (en $\mu \mathrm{m}=10^{-6} \mathrm{~m}$ )

$d p_{50} \quad$ ouverture du tamis qui laisse passer $50 \%$ (en poids) d'une poudre (en $\mu \mathrm{m}=10^{-6} \mathrm{~m}$ )

$D_{D}, D_{E}, D_{S} \quad$ coefficients de dispersion axiale par unité de section et de hauteur lit de la phase dense, du gaz et du solide de la phase dense $\left(\mathrm{m}^{2} / \mathrm{s}\right)$

$D_{D O} \quad$ coefficient axial de dispersion de la phase dense par unité de section de lit et unité de hauteur de phase dense $\left(\mathrm{m}^{2} / \mathrm{s}\right)$

$D_{T} \quad$ diamètre de l'appareil ou du lit fluidisé (en m ou $\mathrm{cm}$ )

$E$ coefficient de dispersion axiale du gaz de la phase dense par unité de section du gaz de la phase dense et de hauteur de lit $\left(\mathrm{m}^{2} / \mathrm{s}\right)$

$E_{o} \quad$ coefficient de dispersion axial : $E / h=E_{o} / h_{o}$

$f_{b}, f_{d}, f_{m} \quad$ fractions volumiques du lit occupées par les bulles, le gaz de la phase dense et le solide $\left(f_{b}+f_{d}+f_{m}=1\right)$

$g$

$h$

$h_{o}$

$h_{m f}$ $h_{r}$

$h_{S}$

$H_{K}, H_{E}, H_{R} \quad$ voir $H_{O K}, H_{O E}, H_{O R}, \beta H_{K}=H_{O K}\left(h / h_{\mathrm{o}}\right)$, $\beta H_{E}=H_{O E}\left(h / h_{o}\right), \beta H_{R}=H_{O R}\left(h / h_{o}\right)$

$H_{O K}, H_{O E}, H_{O R}$ hauteurs d'unité de transfert, de dispersion et de réaction (en hauteurs de phase dense)

$H_{O K}=\left(U-U_{o}\right) / K_{o}, H_{O E}=f_{d} E_{o} /\left(U-U_{o}\right)$, $H_{O R}=\left(U-U_{o}\right) / k_{\mathrm{o}}($ en m $)$

$k \quad$ constante de cinétique chimique (réaction du premier ordre) $k h=k_{o} h_{o}$ (en $1 / \mathrm{s}$ )

$k_{o} \quad$ constante cinétique (réaction du premier ordre) par unité de volume de phase dense (1/s)

$K \quad \operatorname{voir} K_{o}: K h=K_{o} h_{o}($ en $1 /$ s)

$K_{o} \quad$ coefficient de transfert de masse entre le gaz des bulles et celui de la phase dense par unité de volume de phase dense (en 1/s)

$n_{B} \quad$ résultat défini par la relation (3)

$N_{K}, N_{E}, N_{R} \quad \operatorname{voir} N_{O K}, N_{O E}, N_{O R}: N_{K}=\beta \cdot N_{O K}, N_{E}=\beta \cdot N_{O K}$, $N_{R}=\beta \cdot N_{O R}$

$N_{O K}, N_{O E}, N_{O R}$ nombres d'unités de transfert, de dispersion et de réaction pour le lit

$N_{O K}=h_{O} / H_{O K}, N_{O E}=h_{o} / H_{O E}, H_{O R}=h_{o} / H_{O R}$

$R$ fraction de $C_{o}$ transformée chimiquement $R=\left(C_{o}-C_{S}\right) / C_{o}$ constante de temps (voir le texte utilisation d'un gaz traceur) temps (en s) vitesse superficielle du gaz dans le lit (en $\mathrm{m} / \mathrm{s}$ ) 


$\begin{array}{ll}U_{B X} & \begin{array}{l}\text { coefficient d'expansion du lit } h_{o} / U_{B X} \\ \end{array} \\ & \left(h-h_{o}\right) /\left(U-U_{o}\right)(\text { en } \mathrm{m} / \mathrm{s}) \\ U_{m f} & \text { vitesse superficielle du gaz au minimum de } \\ & \text { fluidisation }(\text { en } \mathrm{cm} / \mathrm{s}) \\ & \text { vitesse superficielle du gaz dans la phase } \\ & \text { dense }(\mathrm{en} \mathrm{cm} / \mathrm{s} \mathrm{ou} \mathrm{m} / \mathrm{s}) .\end{array}$

\section{SYMBOLES GRECS}

$\beta$ fraction du débit qui s'écoule par les bulles $\beta=\left(U-U_{o}\right) / U$

$\theta$ temps normé par rapport au temps de séjour moyen $\theta=t / \theta_{m}$

$\theta_{B}$ temps de séjour moyen de la phase bulle dans le lit (en s).

$\theta_{m}$ temps de séjour moyen des gaz dans le lit $\theta_{m}=$ $\left(h-h_{S}\right) / U($ en s)

$\varphi$ fraction du gaz du lit sous forme de bulles $\varphi=$ $\left(h-h_{\mathrm{o}}\right) /\left(h-h_{S}\right)$

$\varepsilon \quad$ fraction du volume du lit occupé par le gaz

$\sigma$ déviation relative standard

$\mu \mathrm{m}$ abréviation de micron $\left(10^{-6} \mathrm{~m}\right)$.

\section{INTRODUCTION}

Après avoir présenté une documentation succincte sur les études, modèles et méthodes relatifs à l'extrapolation, nous détaillerons quelques travaux conduits avec des maquettes et des pilotes. Les performances qu'il est possible d'atteindre et les règles à suivre pour les obtenir en seront déduites.

\section{1 ÉTUDES, MODÈLES, MÉTHODES D'EXTRAPOLATION}

Au préalable, nous rappellerons quelques phénomènes de base pour situer le domaine des lits fluidisés catalytiques. Les travaux d'extrapolation publiés seront résumés. Nous donnerons ensuite quelques éléments sur les lits fluidisés rapides, qui sont leur prolongement. Enfin, l'examen des analogies de comportement entre les systèmes gaz-solide à particules fines, auxquels les lits catalytiques appartiennent, et les systèmes gaz-liquide terminera ce chapitre.

\subsection{Phénomènes de base - Domaine des lits catalytiques}

Considérons un tube de $0,1 \mathrm{~m}$ de diamètre, susceptible d'être traversé par un courant de gaz uniformément réparti sur toute sa section par une plaque poreuse. Plaçons sur cette plaque une couche de 0,1 m de support de catalyseur de craquage catalytique, dont le diamètre moyen des particules est de l'ordre de 70 microns. Soumettons cette couche à un courant de gaz ascendant croissant. Lorsque la perte de charge du gaz à travers la couche atteint une valeur égale à la pression que la couche exerce sur la plaque poreuse, la couche devient fluide comme un liquide. On peut y plonger un objet.
Si nous continuons à augmenter le débit de gaz, la perte de charge n'augmente plus. Des bulles de plus en plus nombreuses apparaissent au bas du lit. Ces bulles éclatent à sa surface en projetant des particules, dont une partie est entraînée par le courant gazeux. Dans les grands appareils, des vitesses de gaz de $0,5 \mathrm{~m} / \mathrm{s}$ et même $0,7 \mathrm{~m} / \mathrm{s}$ sont possibles. Les particules entraînées sont captées avec des cyclones et recyclées (Fig. 1). L'ensemble de ces phénomènes est appelé fluidisation. La vitesse superficielle du gaz $U_{m f}$ lorsque les particules deviennent mobiles est la vitesse minimum de fluidisation.

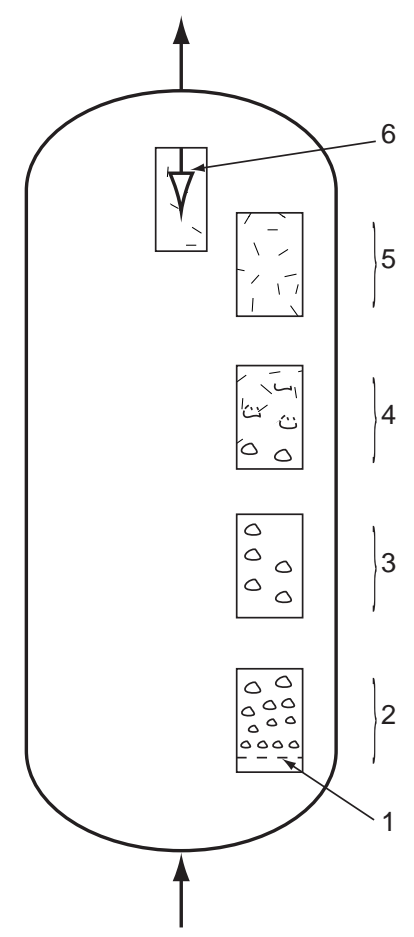

Figure 1

Constitution d'un lit fluidisé catalytique. 1 : grille de répartition du gaz. 2 : formation des bulles. $3:$ lit dans lequel on peut immerger un échangeur de chaleur (tubes verticaux). 4 : sommet du lit, éclatement des bulles et projection de catalyseur (zone de désengagement). 5 : entraînement de catalyseur. 6 : séparation du gaz du catalyseur et retour du catalyseur dans le lit ( 3 cyclones en série).

A catalytic fluidized bed design. 1: distributor of the gas. 2: formation of bubbles. 3: fluidized bed where it is possible to immerse heat transfer tubes. 4: top of the bed, splitting of bubbles with projection of catalyst (transport disengaging height). 5: carry over of catalyst. 6: gas-catalyst separators (3 stages of cyclones) and go back of catalyst into the bed (diplegs).

Le tableau 1 montre comment Umf évolue en fonction du diamètre dp des particules lorsque leur densité apparente est égale à 1 et que le gaz est de l'air. 
TABLEAU 1

\begin{tabular}{l|l|l|l|l|l}
\hline$d p$ (en micron) & 50 & 150 & 500 & 1000 & 5000 \\
\hline$U_{m f}($ en $\mathrm{cm} / \mathrm{s})$ & 0,12 & 1,0 & 7,7 & 22,4 & 105 \\
\hline
\end{tabular}

Le comportement décrit ci-dessus varie avec la dimension des particules. Matheson et al. (1949) ont donné les premiers points de repère pour des particules de densité apparente égale à 1 :

- pour $d p<10$ microns : il existe de fortes cohésions entre les particules. La poudre n'est pas fluidisable ;

- pour $d p \cong 25$ microns : le gaz se crée des passages préférentiels (renardages) qui disparaissent quand on augmente le débit de gaz;

- pour $d p \cong 45$ microns : les forces de cohésion ont moins d'effet. L'obtention d'une très belle fluidisation est possible. Les renardages ont disparu et les bulles sont petites. C'est ce qui convient aux lits catalytiques à hautes performances ;

- pour $d p \cong 150$ microns : les bulles sont très grosses. De fortes vibrations apparaissent.

Geldhart (1973) a classé les poudres en quatre groupes (A, $\mathrm{B}, \mathrm{C}, \mathrm{D})$ en prenant en compte le diamètre moyen $d p$ des particules et leur densité apparente $d a_{p}$. Les catalyseurs performants des unités industrielles se situent dans le groupe A $\left(25<d p<225\right.$ microns pour $\left.d a_{p}=1\right)$, près du groupe $\mathrm{C}$ $\left(d p<25\right.$ microns pour $\left.d a_{p}=1\right)$.

\subsection{Problèmes relatifs au contact gaz-catalyseur}

Supposons que la poudre soit un catalyseur et le gaz un mélange réactionnel. Le lit fluidisé devient un réacteur chimique dont on peut, en principe, calculer les performances, si les équations cinétiques du système réactionnel sont connues et si l'on sait caractériser quantitativement comment s'effectue le contact entre les réactifs et le catalyseur. Malheureusement, les lois de mise en contact ne sont pas accessibles pour les lits fluidisés, comme elles le sont pour les lits fixes, les colonnes à garnissage, etc.

Le paramètre géométrique qui gère le contact gaz-solide dans les lits fluidisés industriels est le diamètre des bulles. Sa valeur varie largement et l'expérience montre qu'il est impossible de la relier d'une façon simple à celle des particules de la poudre fluidisée, comme dans les lits fixes à celle des grains de catalyseurs ou dans les colonnes à garnissage à celle des éléments de remplissage.

Pour obtenir des bulles dont la taille permet d'avoir des réacteurs performants, les particules à utiliser sont d'une finesse telle que les forces d'adhésion entre les particules ne sont pas négligeables par rapport à leurs poids. Or, on ne sait pas quantifier ces forces d'adhésion, qui sont souvent fonction de l'environnement physicochimique des particules. Des associations entre particules se forment et de ce fait, une poudre peut donner des bulles de dimensions très différentes selon qu'elle est fluidisée par de l'air ou par un milieu réactionnel complexe, dans lequel le solide joue le rôle de catalyseur.
La taille des bulles dans un réacteur industriel est, même dans les meilleurs cas (5-6 cm en visuel), grande par rapport au diamètre des appareils de laboratoire. Il est de ce fait impossible de réaliser en laboratoire des conditions de mise en contact identiques à celles rencontrées dans un réacteur industriel. En laboratoire, la taille des bulles est, à peu de chose près, imposée par le diamètre de l'appareil. Ceci désensibilise le chimiste vis-à-vis de l'importance du phénomène bulles. De plus, s'il essaie d'optimiser la granulométrie, il trouve qu'à quantité de catalyseur constante, les lits à particules grossières sont plus performants que ceux à particules fines. Ce fait l'incite à s'orienter vers les grosses particules lors de l'extrapolation, or, c'est l'inverse qu'il faut faire.

Quel est, a priori, le diamètre d'un pilote dans lequel les bulles peuvent se développer librement afin que l'extrapolation puisse être faite avec une hauteur de lit constante et en conservant les performances chimiques ? Si l'on admet que cette condition est satisfaite lorsque le diamètre du pilote égale 5 à 10 fois celui des bulles, le diamètre du pilote à envisager doit, dans les meilleurs cas (bulles de $5 \mathrm{~cm}$ ), être de 0,25 à $0,50 \mathrm{~m}$. Alimenté avec une vitesse de gaz de $0,5 \mathrm{~m} / \mathrm{s}$, le pilote devient alors un petit atelier de production. Investissement et exploitation ne sont possibles qu'en milieu industriel et ne se justifient que pour des produits à très gros tonnage.

Les bulles entraînent dans leur sillage du catalyseur vers le haut du lit. Lorsqu'il redescend, il entraîne du gaz. Ce recyclage du gaz est défavorable pour la chimie. Par contre, cette circulation du solide génère deux propriétés très appréciées : une grande homogénéité de température et un coefficient de transfert de chaleur élevé avec toute surface immergée.

\subsection{Extrapolation : méthodes et travaux publiés}

\subsubsection{Le premier atelier de craquage catalytique}

Pour fabriquer des essences à partir de distillats plus lourds, Houdry avait élaboré un procédé catalytique à lit fixe dès 1936. Lewis, professeur au MIT, a conseillé à Esso en 1940 d'améliorer ce procédé en opérant avec des particules fines de catalyseur (80 microns) en suspension. Carlsmith et Johnson de Esso Laboratories, Standard Oil Company ont décrit en 1945 le déroulement de la mise au point du premier atelier de craquage catalytique qui en a résulté.

Le procédé requiert un réacteur pour le craquage et un réacteur pour la régénération du catalyseur. Il a été élaboré et extrapolé à partir d'un appareil de laboratoire, en mettant en œuvre deux pilotes et deux maquettes. La mission du laboratoire était d'établir la chimie, celle des deux pilotes a été :

- de déterminer les conditions opératoires pour obtenir les mêmes produits qu'en laboratoire ;

- d'obtenir toutes les données nécessaires pour la construction de l'unité d'exploitation ;

- de fournir des produits pour des essais d'application. 
Le catalyseur était introduit avec les réactifs et séparé d'eux dans des cyclones.

Les objectifs des maquettes ont été :

- pour l'une, d'étudier la circulation du solide et de former le personnel de l'unité future d'exploitation ;

- pour l'autre, de définir la granulométrie du catalyseur en examinant ses aptitudes à la fluidisation.

L'extrapolation fut un succès. Le réacteur de craquage avait $5 \mathrm{~m}$ de diamètre. Dans le deuxième atelier, le catalyseur sortait des réacteurs par des déversoirs et non par les cyclones. L'ensemble a été réalisé en deux ans. En 1945, trente-cinq ateliers fonctionnaient.

\subsection{2 À la recherche d'un modèle phénoménologique}

Disposer d'un modèle permet de classer tous les résultats d'essai et de mieux formuler les tendances à suivre pour améliorer les performances. S'il représente correctement les faits et si l'on connaît les valeurs de ses paramètres, il permet de concevoir un réacteur pour tout système réactionnel dont les équations cinétiques sont établies.

Certains aspects de l'hydrodynamique et du contact gazcatalyseur ont été examinés avec des techniques de traceurs. Elles ont été utilisées en laboratoire par Gilliland et al. (1949, $1952,1953)$ et sur des unités industrielles de craquage catalytiques par Danckwerts et al. (1954), Handlos et al. (1957), Singer et al. (1957). D'après ces travaux, on peut tirer les conclusions suivantes :

- l'écoulement du gaz à travers le lit fluidisé est beaucoup plus proche d'un écoulement de type piston que parfaitement mélangé. Le rétromélange représente moins de $10 \%$ du débit total (d'après Danckwerts et al.) ;

- le solide est complètement mélangé (selon Singer et al.);

- le gaz présent dans la phase dense n'est pas complètement mélangé (selon Handlos et al.);

- le contact entre le gaz et le solide est imparfait. L'avancement effectif d'une réaction du premier ordre est très inférieur à celui calculé avec la courbe de la répartition des temps de séjour du gaz (d'après Gilliland et al.). Ce résultat exige d'établir un modèle.

Des modèles ont ensuite été proposés par Shen et Johnstone (1954), Mathis et Watson (1956), Lewis et Gilliland (1959) et May (1959). Tous admettent que le lit est constitué d'une phase bulles, par laquelle s'écoule la majorité du gaz selon le mode piston, en mélangeant intensément une suspension de catalyseur appelée phase dense, dont l'aspect rappelle celui d'un lit fluidisé au voisinage de sa vitesse minimum de fluidisation. Leurs différences portent sur l'état de mélange du gaz de la phase dense (totalement, partiellement ou non mélangé) et sur la présence ou non de catalyseur dans la phase bulles.

Le modèle de May (1959) exclut la présence de catalyseur dans la phase bulles. Il caractérise le mélange du gaz de la phase dense par un coefficient de dispersion axial et le contact entre le gaz de la phase bulles et le catalyseur par un coefficient de transfert de masse. C'est un modèle à deux paramètres. Leurs valeurs, mesurées en fonction des conditions opératoires (dimensions du lit, débits de gaz, granulométries, etc.), indiqueront les tendances à suivre pour obtenir de bonnes performances.

D'après les publications, au moins deux sociétés après Esso (avec laquelle May travaillait) se sont aidées de ce modèle pour industrialiser une réaction : Shell et Péchiney Saint-Gobain (PSG). Les travaux de Shell ont été relatifs au procédé Deacon (réaction équilibrée de transformation de l'acide chlorhydrique en chlore par oxydation à l'air), alors que ceux de $P S G$ ont concerné un procédé d'oxychloration de l'éthylène en dichloréthane, dans des conditions non sélectives en vue de générer simultanément d'autres dérivés chlorés. Des pilotes de 0,5 $\mathrm{m}$ de diamètre ont été nécessaires. Les extrapolations ont été des succès (taux de transformations $>99 \%$ ).

Une analyse détaillée des études publiées par ces sociétés est présentée ci-après. Les valeurs des paramètres données dans ces publications sont comparées, ainsi que les conditions dans lesquelles elles ont été obtenues (granulométrie, vitesse des gaz, diamètre de l'appareil, etc.). On peut en déduire que de très hautes performances par passe sont réalisables et formuler quelques règles à suivre. En complément, Botton (1980), dix ans après avoir extrapolé le procédé $P S G$ d'oxychloration non sélective de l'éthylène, a signalé que ce modèle permettait de bien représenter les résultats expérimentaux, même pour des réactions complexes.

Ces études phénoménologiques ont seulement généré un modèle correct et des règles de tendances à respecter pour faire évoluer un procédé vers des performances de plus en plus élevées. Cependant, aucune corrélation véritable entre les valeurs mesurées des paramètres et les conditions opératoires n'a été établie. Ce type de corrélation impliquerait que l'on puisse caractériser une poudre au moyen d'un paramètre, deux au plus. Il est loin d'être évident que ceci soit possible pour les poudres de particules fines.

\subsubsection{Les modèles mécanistiques à bulles}

Le lit est supposé constitué de bulles identiques, qui montent à travers la phase dense en conservant leur identité de bas en haut du lit. Des modèles d'échange entre bulles et phase dense ont été proposés par Davidson et Harrison (1963), Patridge et Rowe (1966), puis Kunii et Levenspiel (1968). Les échanges sont supposés s'effectuer par un mécanisme de diffusion et par une circulation de gaz. Cette circulation pénètre au fond de la bulle et sort à son sommet. Cet échange est négligeable si les particules sont fines.

Cette image des lits fluidisés est proche de ce que l'on observe dans des lits à particules grossières, à faibles débits de gaz, où les bulles sont de géométrie bien définie. C'est très 
éloigné de ce que l'on voit dans les lits à particules fines à haute vitesse de gaz, seul domaine utilisable pour les lits catalytiques à haute performance. Dans ce cas, les bulles n'ont aucune forme géométrique définie, mais coalescent entre elles et se brisent en permanence.

Les relations proposées permettent de quantifier l'hydrodynamique du lit et le contact gaz-solide dès que l'on a fixé le diamètre des bulles. Ce sont des modèles à un paramètre : le diamètre des bulles. Des écritures très sophistiquées ont été proposées par plusieurs auteurs. On peut noter celle de Werther (1980) qui introduit pour les transferts des analogies avec les milieux gaz-liquide. Sergiou et Laguerie (1983) ont comparé quatre d'entre elles, pour la réaction d'amoxydation du propylène en acrylonitrile dans un appareil de laboratoire.

Ces modèles mécanistiques à bulles ne dispensent cependant pas d'exploiter un pilote de $0,5 \mathrm{~m}$ de diamètre pour acquérir la certitude que le catalyseur élaboré est apte à donner d'excellentes performances. La situation est différente avec des particules grossières pour lesquelles la cartographie des bulles est, pour une géométrie du lit donnée, indépendante de la granulométrie (cf. étude du phénomène bulles, dans la deuxième partie de cet article). Mais ces lits n'ont pas les performances exigées par les lits catalytiques de l'industrie chimique.

\subsubsection{Modèle phénoménologique à $U_{m f}(1980)$ : ses conséquences}

Plus tardivement, en 1980, Botton a montré avec des poudres et des gaz inertes que toutes les propriétés d'un lit pouvaient être corrélées en fonction de la vitesse minimum de fluidisation. Les corrélations établies couvrent l'hydrodynamique et le contact gaz-solide (les paramètres de May), aussi bien pour des appareils de laboratoire (lits à bulles gênées ou pistonnantes) que pour des appareils industriels (lits à bulles libres). Le modèle phénoménologique à deux paramètres de May devient un modèle à un paramètre : la vitesse minimum de fluidisation $U_{m f}$ Il en serait de même pour tout autre modèle phénoménologique pertinent.

Que peut-on attendre de ce modèle à un paramètre sachant que celui de May représente bien les faits expérimentaux pour des systèmes chimiques complexes?

- Que toute propriété physique mesurée en laboratoire sur un

catalyseur en milieu réactionnel permet, avec les corrélations établies en milieu inerte, de lui attribuer une valeur de vitesse minimum de fluidisation dite "de comportement" dans l'environnement où il se trouve. Adoptons cette notion de " $U_{m f}$ de comportement" et le modèle de May pour caractériser un lit.

Une valeur de $U_{m f}$ dite "de comportement" étant choisie, les relations établies en milieu inerte autorisent le calcul, avec le modèle de May, des performances d'un réacteur fictif industriel et d'un réacteur de laboratoire aux performances identiques ou proches. Le réacteur de laboratoire devient donc une simulation du réacteur industriel. Le problème est de réaliser un catalyseur susceptible d'assurer cette valeur de $U_{m f}$ de comportement choisie, pour obtenir les performances souhaitées.

Ainsi ce modèle ouvre les perspectives suivantes :

- possibilité de cerner les propriétés d'une poudre avec un seul paramètre ;

- des méthodes simples pour améliorer les catalyseurs ;

- possibilités d'extrapolation directe, sans pilote ;

- possibilité d'utiliser cette technique pour des produits à faible tonnage.

Nous développerons ci-dessous en détail comment ce modèle a été élaboré, et de quelle façon l'utiliser.

\subsubsection{Lits fluidisés rapides}

Les lits fluidisés catalytiques travaillent à des vitesses de gaz maximums de 0,6 à $0,8 \mathrm{~m} / \mathrm{s}$. Il est logique, pour améliorer les productivités, de chercher comment les augmenter. Lewis et Gilliland ont, d'après Yerushalmi et al. (1976), clairement identifié dès 1940 ce que l'on appelle un lit fluidisé rapide. Ils ont noté qu'aux vitesses de gaz suffisantes (>1,5 m/s), pour évacuer dans un temps très court tout le catalyseur d'un réacteur, on obtenait, en injectant du catalyseur en continu, un lit stabilisé dont la rétention en solide était fonction du débit injecté et de la vitesse des gaz. Le premier atelier de craquage catalytique (voir ci-dessus) était un lit fluide rapide. D'après Reh (1971), Lurgi a élaboré deux procédés en lit fluidisé rapide : la calcination de l'alumine hydratée en alumine métallurgique et l'absorption de l'acide fluorhydrique qui se dégage des cuves d'électrolyse de l'aluminium.

Les lits fluidisés rapides fonctionnent à des vitesses de gaz de 2,5 à $6 \mathrm{~m} / \mathrm{s}$ et des teneurs en solide de 10 à $20 \%$ en volume. Les capacités de production sont élevées et, malgré leur faible teneur en solide, ils possèdent d'excellentes propriétés : l'écoulement du gaz est de type piston, le contact gaz-solide est bon, les transferts de chaleur sont excellents et la température est uniforme. Kunii et Levenspiel (1993) leur ont consacré un chapitre dans leur ouvrage et une session lors du $8^{e}$ Congrès international sur la fluidisation (Tours, mai 1995). Ils font d'ailleurs régulièrement l'objet de congrès internationaux.

\subsubsection{Analogies entre les systèmes gaz-solide et gaz-liquide}

Lorsqu'on injecte dans un liquide du gaz à des vitesses très supérieures à celles utilisées dans les colonnes à bulles classiques, on constate de bas en haut : une zone à concentration constante (émulsion), suivie d'une zone à concentration décroissante (zone vésiculaire) et enfin une zone à concentration constante (entraînement saturé). Cette image est identique à celle que l'on a pour les lits fluidisés : le lit lui-même (émulsion), la zone de désengagement (zone vésiculaire) et celle d'entraînement à saturation (entraînement saturé).

Avidan et Yerushalmi (1982) ont mesuré dans une colonne de $0,15 \mathrm{~m}$ de diamètre et de $8,5 \mathrm{~m}$ de hauteur la fraction de vide $\varepsilon$ d'un lit fluidisé de particules fines (FCC-diamètre 
moyen des particules de $49 \mu \mathrm{m}$ ) en fonction de la vitesse $U$ des gaz $(0,1$ à $8 \mathrm{~m} / \mathrm{s})$ et du débit de solide $G s$ en circulation (de 50 à $\left.150 \mathrm{~kg} / \mathrm{m}^{2} \cdot \mathrm{s}\right)$. Gs n'a de l'effet que pour $U>1,5 \mathrm{~m} / \mathrm{s}$. Du graphique obtenu en portant $\log (\varepsilon)$ en fonction de $\log (U)$ ils ont déduit :
$\varepsilon<0,65$
lit bullant, $U<0,4 \mathrm{~m} / \mathrm{s}$
$0,65<\varepsilon<0,80$
lit turbulent, $0,4<U<1,3 \mathrm{~m} / \mathrm{s}$
$0,8<\varepsilon<0,945$
lit fluidisé rapide,
$1,3<U<2,2 \mathrm{~m} / \mathrm{s}$ si $G s=50 \mathrm{~kg} / \mathrm{m}^{2} \cdot \mathrm{s}$
$1,3<U<5,5 \mathrm{~m} / \mathrm{s}$ si $G s=150 \mathrm{~kg} / \mathrm{m}^{2} \cdot \mathrm{s}$
$0,945<\varepsilon<0,99$ transport pneumatique dense

$$
\begin{aligned}
& 2,2<U<4 \mathrm{~m} / \mathrm{s} \text { si } G s=50 \mathrm{~kg} / \mathrm{m}^{2} \cdot \mathrm{s} \\
& 5,5<U<12 \mathrm{~m} / \mathrm{s} \mathrm{si} G s=150 \mathrm{~kg} / \mathrm{m}^{2} \cdot \mathrm{s}
\end{aligned}
$$

$\varepsilon>0,99$

transport pneumatique dilué.

Botton et al. (1978) ont expérimenté dans une colonne de $0,075 \mathrm{~m}$ de diamètre et de $3,2 \mathrm{~m}$ de hauteur avec de l'air et de l'eau. Ils ont mesuré pour $0,05<U<15 \mathrm{~m} / \mathrm{s}$ les teneurs en eau de l'émulsion, de la zone vésiculaire et de celle d'entraînement en saturation. Pour remplir la colonne d'émulsion, le débit de liquide injecté doit être supérieur à celui entraîné à saturation, afin que la sortie de la colonne soit située dans la zone vésiculaire. Du graphique obtenu $\{\log (1-\varepsilon)$ en fonction de $\log (U)\}$ ils ont déduit pour la zone émulsion :

$$
\begin{array}{ll}
U<0,2 \mathrm{~m} / \mathrm{s} & \varepsilon<0,3 \text {, écoulement à petites bulles } \\
0,2<U<1,35 \mathrm{~m} / \mathrm{s} & \begin{array}{l}
0,3<\varepsilon<0,56, \text { formation de grosses } \\
\text { bulles }
\end{array} \\
1,35<U<5,5 \mathrm{~m} / \mathrm{s} & \begin{array}{l}
(0,56<\varepsilon<0,85) \text {, évolution d'une } \\
\text { dispersion de gaz dans un liquide à } \\
\text { celle d'un liquide dans un gaz }
\end{array} \\
5,5<U<13,5 \mathrm{~m} / \mathrm{s} & \begin{array}{l}
(0,85<\varepsilon<0,98), \text { lit de gouttes } \\
\text { transportées } \\
U>13,5 \mathrm{~m} / \mathrm{s}
\end{array} \\
& \text { dispersion uniforme en petites gouttes. }
\end{array}
$$
$5,5<U<13,5 \mathrm{~m} / \mathrm{s} \quad(0,85<\varepsilon<0,98)$, lit de gouttes transportées

Quand $U$ croît, la teneur de la zone émulsion diminue et celle de la zone d'entraînement augmente. À $U=13,5 \mathrm{~m} / \mathrm{s}$ elles s'égalisent. $U=13,5 \mathrm{~m} / \mathrm{s}$ est une vitesse critique au-delà de laquelle le liquide se disperse uniformément sous la forme de petites gouttes.

Le contact gaz-liquide dans la zone relative au lit à gouttes transportée est très efficace, même trop efficace. Il est préconisé pour des arrêts de gaz polluant et de poussières (Botton et al., 1997) d'opérer, pour des raisons d'économie d'énergie, à $U=10 \mathrm{~m} / \mathrm{s}$ avec un débit de liquide de 10 à $15 \mathrm{~kg} / \mathrm{m}^{2} \cdot \mathrm{s}$, ce qui est très inférieur aux entraînements saturés $\left(60 \mathrm{~kg} / \mathrm{m}^{2} \cdot \mathrm{s}\right)$.

Les analogies entre les deux systèmes sont illustrées sur la figure 2 , qui représente pour chacun d'eux $1 /(1-\varepsilon)$ en fonction de $U$. La courbe relative au système gaz-solide est déduite des travaux de Avidan et Yerushalmi (1982) pour des débits élevés de particules, alors que celle du système gaz-liquide correspond aux résultats de Botton et al. (1978) relatifs aux zones

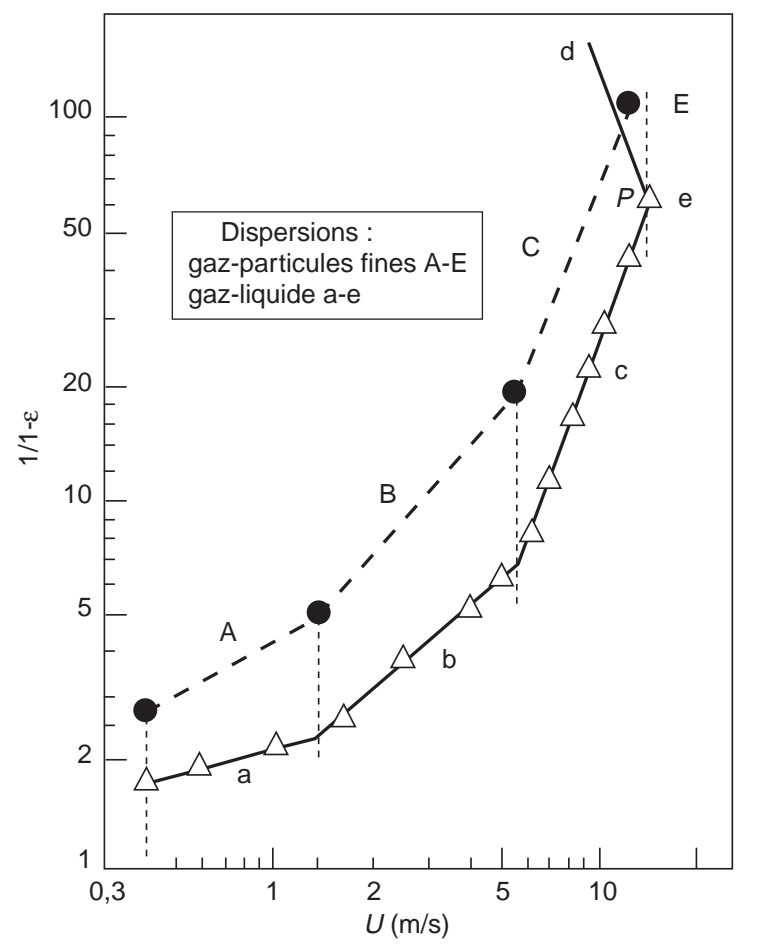

Figure 2

Évolution de la porosité $\varepsilon$ : représentation de $1 /(1-\varepsilon)$ en fonction de la vitesse superficielle des gaz $U$, pour des systèmes gaz-particules fines et gaz-liquide (établie à partir des courbes d'Avidan et Yerushalmi (1982) à haut débit de particules et de Botton et al. (1978) relatives aux zones d'émulsion et d'entraînement pour des systèmes gaz-liquide). A : lits fluidisés turbulents ; B : lits fluidisés rapides ; C : transports pneumatiques denses ; E : pour $U_{G}>12 \mathrm{~m} / \mathrm{s}$ transports pneumatiques dilués. a : zones d'émulsion à grosses bulles ; b : de transition; $\mathrm{c}:$ des lits à gouttes transportées ; d : zone d'entraînement ; e : pour $U_{G}>13,5 \mathrm{~m} / \mathrm{s}$ dispersion uniquement en fines gouttelettes.

Variation of porosity $\varepsilon$ plotted $1 /(1-\varepsilon)$ against superficial velocity $U$ of gas for gas-fine particles system (deduced from Avidan and Yerushalmi (1982) data at high flow of particles) and for gas-liquid system (deduced from Botton et al. (1978) for emulsions and carry over zones). A: turbulent fluidized bed; B: fast fluidized bed; C: high-density pneumatic transport. E: for $U_{G}>12 \mathrm{~m} / \mathrm{s}$ low-density pneumatic transport. a: large bubbles emulsion; $b$ : transition zone; $c$ : dops transported bed zone; $d$ : carry over zone; $e$ : for $U_{G}>13.5 \mathrm{~m} / \mathrm{s}$ only very fine drops dispersion is obtained.

d'émulsion et d'entraînement. On peut relever sur cette figure que les vitesses du gaz, pour lesquelles on assiste à des changements de régime dans chacun des systèmes, sont très voisines. Leurs valeurs sont très proches de : $1,3-5,5$ et $13 \mathrm{~m} / \mathrm{s}$.

Les fractions de vide à $1,3 \mathrm{~m} / \mathrm{s}$ sont respectivement $\varepsilon=0,80$ et 0,56 . Elles deviennent très voisines si l'on prend pour le lit fluidisé le taux de vide par rapport à sa phase dense et non par rapport au solide. En effet, on obtient, en supposant que la porosité de la phase dense est de $0,5: \varepsilon=0,60$ et 0,56 . La même remarque est valable pour $U=5,5 \mathrm{~m} / \mathrm{s}$. La vitesse critique $U=13 \mathrm{~m} / \mathrm{s}$ rappelle la température critique 
des systèmes liquide-vapeur. Au-delà, les systèmes sont de type monophasique : uniquement de la vapeur, des petites gouttes ou des particules bien dispersées.

\section{EXEMPLES DE TRAVAUX D'EXTRAPOLATION AVEC UN PILOTE}

Les exemples de travaux que nous donnons utilisent le modèle de May (1959). Nous décrirons ce modèle en détail, résumerons quelques méthodes de mesure de ses paramètres, exposerons les résultats publiés par les expérimentateurs puis, nous en déduirons les règles de tendance à suivre pour extrapoler ou améliorer les performances d'un catalyseur.

\subsection{Modèle de May (1959) - Formulation}

\subsubsection{Hypothèses et commentaires}

Les hypothèses de base du modèle de May sont :

- le lit est constitué d'une phase bulles et d'une phase dense ;

- la phase dense contient la totalité du catalyseur ;

- la majorité du gaz s'écoule par la phase bulles en régime piston et la fraction restante par la phase dense en régime mélangé ;

- le volume de la phase dense et le débit de gaz qui y transite sont supposés indépendants du débit total de gaz et égaux à ce qu'ils sont lors de l'apparition des premières bulles à la mise en fluidisation ;

- le contact entre le gaz et le solide dans la phase dense est supposé identique à ce qu'il serait dans un lit fixe constitué avec les mêmes grains, donc parfait. Si les bulles contiennent un peu de catalyseur, celui-ci n'aura d'effet que si la réaction chimique est très rapide, cas dans lequel l'émulsion est très appauvrie en réactif frais.

Ces éléments étant admis, deux coefficients suffisent pour caractériser le contact gaz-solide : un coefficient de transfert entre le gaz de la phase bulles et le gaz de la phase dense, et un coefficient axial de mélange du gaz de la phase dense. May les suppose constants dans tout le lit.

Ce modèle à deux paramètres (les deux coefficients) paraît simpliste. Il néglige les effets des zones proches du distributeur (formation des bulles), du sommet du lit (éclatement des bulles et catalyseur entraîné) et des parois (effet Gulf Stream). Les résultats expérimentaux ont montré (on le verra ci-après) que les effets de grille et de sommet de lit peuvent être séparés de ceux du lit par une étude en fonction de la hauteur du lit. Les effets de parois sont, par contre, liés au diamètre du lit.

L'expérience montre qu'il est conseillé pour les premières mesures des deux coefficients de ne porter son attention que sur leurs valeurs moyennes dans le lit. En revanche, leur évolution avec les conditions opératoires indiquent les tendances à suivre pour les améliorer.

\subsubsection{Nomenclature et mise en équation}

La nomenclature donnée ici (introduite par Botton, 1970) se réfère au volume de phase dense par unité de section de lit (hauteur de phase dense). Elle est indépendante de la vitesse du gaz.

- $h, h_{o}, h s$ (en m) : les volumes par unité de section (appelées hauteurs) respectivement du lit, de la phase dense et du solide (à porosité nulle).

$-f_{b}, f_{d}, f_{m}$ (sans dimension) : les fractions volumiques du lit occupées par les bulles, le gaz de la phase dense et le solide. D'où $f_{b}=\left(h-h_{o}\right) / h, \quad f_{d}=\left(h_{o}-h_{S}\right) / h, \quad f_{m}=h_{s} / h$.

- $U, U_{o},\left(U-U_{o}\right)($ en $\mathrm{m} / \mathrm{s})$ : les débits par unité de section du lit (appelées vitesses superficielles) du gaz qui s'écoule à travers le lit, la phase dense et la phase bulles.

$-K_{o}\left(\right.$ en s $\left.^{-1}\right)$ : le coefficient de transfert entre la phase bulles et le gaz de la phase dense par unité de volume de phase dense. $K_{o} \cdot h_{o}$ est le débit total transféré dans le lit, et $N_{O K}=$ $K_{o} \cdot h_{o} /\left(U-U_{o}\right)$, le nombre d'unités de transfert du lit.

- $E_{o}\left(\right.$ en $\left.\mathrm{m}^{2} \cdot \mathrm{s}^{-1}\right)$ : le coefficient de diffusion axiale du gaz de la phase dense. $f_{d} E_{o} / h_{o}$ représente le débit de gaz par unité de section de lit recyclé du haut en bas du lit. $N_{O E}=\left(U-U_{o}\right)$ $h_{o} / f_{d} E_{o}$, le nombre d'unités de diffusion axiale du lit.

- $k_{o}\left(\right.$ en $\left.^{-1}\right)$ : la constante cinétique d'une réaction chimique du premier ordre (sans variation de volume) par unité de volume de phase dense. $N_{O R}=k_{o} \cdot h_{o} /\left(U-U_{o}\right)$ est le nombre d'unités de réaction.

- Les hauteurs de phase dense $H_{O K}, H_{O E}, H_{O R}$, qui correspondent à $N_{O K}=1, N_{O E}=1, N_{O R}=1$, sont les hauteurs d'unité de transfert, de diffusion et de réaction.

Ainsi, le nombre d'unités de transfert du lit est égal au rapport entre le débit total de transfert entre les bulles et le gaz de la phase dense, et le débit qui s'écoule par la phase bulles. La hauteur d'unités de transfert est aussi la hauteur de phase dense qu'une bulle doit traverser pour échanger avec le gaz de la phase dense un volume égal à son volume. Des commentaires similaires peuvent être faits pour les autres grandeurs introduites à partir des relations qui les définissent.

Les équations du modèle sont obtenues en effectuant les bilans du réactif (ou du gaz traceur) dans la phase bulles et la phase dense sur un élément différentiel de la hauteur du lit, avec les conditions limites observées ci-après.

- À l'entrée du lit, la concentration dans la phase bulles est prise égale à celle du gaz alimentant le réacteur, et celle dans la phase dense est calculée en tenant compte du débit apporté par le lit par dispersion ;

- au sommet du lit, la concentration dans le gaz de la phase dense est supposée stabilisée.

Une écriture en termes sans dimension est obtenue en normant les concentrations par rapport à la concentration initiale, la hauteur de l'élément différentiel par rapport à la hauteur totale du lit, et le temps (si le régime est transitoire) par rapport au temps de séjour moyen du gaz dans le lit. Les coefficients $K_{o}, E_{o}$, et $k_{o}$ (en cas de réaction chimique) sont incorporés dans les nombres sans dimension $N_{O K}, N_{O E}, N_{O R}$. Ces écritures font apparaître dans les équations deux termes : $-\beta=1-U / U_{o}$, égal à la fraction de gaz qui s'écoule par la phase dense ; 
- si le régime est transitoire (avec un gaz traceur), $\varphi=$ $\left(h-h_{o}\right) /\left(h-h_{s}\right)$ égal à la fraction de gaz présent dans le lit sous forme de bulles.

Une écriture très peu différente a été proposée par Van Deemter (1961). Il néglige $U_{o}$ devant $U\left(U_{o}=0\right)$ et prend pour références $h$ et $U$, et non pas $h_{o}$ et $\left(U-U_{o}\right)$. À $K_{o}$ et $k_{o}$ correspondent :

$K$ et $k$, tels que $K_{o} \cdot h_{o}=K \cdot h, k_{o} \cdot h_{o}=k \cdot h$

À $N_{O K}, N_{O R}, N_{O E}$ correspondent :

$N_{K}=\beta \cdot N_{O K}, N_{R}=\beta \cdot N_{O K}, N_{O E}=N_{E} / \beta$

À $H_{O K}, H_{O R}, H_{O E}$ correspondent :

$H_{K}, H_{R}, H_{E}$, tels que $H_{K}=h / N_{K}, H_{R}=h / N_{R}, H_{E}=h / N_{E}$.

\subsection{Méthodes de mesure des deux paramètres du modèle de May}

\subsubsection{En utilisant une réaction chimique du premier ordre}

Cette méthode permet de mesurer $K_{o}$, donc de connaître $N_{O K}=K_{o} h_{o} /\left(U-U_{o}\right)$ et $H_{O K}$.

May (1959) a donné une solution analytique des équations dans le cas d'une réaction du premier ordre. Une représentation graphique issue de celle proposée par Botton (1970) est donnée figure 3 (pour $\beta=1$ ). Cette figure représente :

$-\log _{10}(1-R)=2,3 \mathrm{Ln}\left(C_{o} / C_{s}\right)$ en fonction de $N_{O R}$ avec en réseau $N_{O K} / N_{O R}$ pour plusieurs valeurs de $N_{O K} / N_{O E}$.
$-N_{O R}=k_{o} \cdot h_{o} /\left(U-U_{o}\right)$ représente le temps de passage du gaz à travers la phase dense normé par rapport au temps de réaction $1 / k_{o}$;

$-N_{O K} / N_{O R}=K_{o} / k_{o}$ représente la vitesse de transfert normée par rapport à celle de la réaction ;

- $N_{O K} / N_{O E}$ à $N_{O K} / N_{O R}=K_{o} / k_{o}$ constant donne l'effet du mélange pour une vitesse de transfert donnée normée par rapport à la vitesse de réaction.

Les travaux de May (1959) et ceux de Gilliland (1949) interprétés par Van Deemter (1961) conduisent à $N_{O K} / N_{O E}<1$. Ces zones ont été ombrées sur la figure 3. Elles montrent que l'effet du mélange du gaz de la phase dense est négligeable sur l'avancement de la réaction. Le graphique se réduit à un faisceau de droites dont l'une représente un réacteur piston idéal ( $N_{O K}$ et $N_{O E}$ infinis) et à la courbe du réacteur idéal mélangé $\left(N_{O K}\right.$ infini et $\left.N_{O E}=0\right)$. La partie utile du graphique donne la relation :

$$
\operatorname{Ln} C_{o} / C_{s}=\operatorname{Ln}[1 /(1-R)]=-\operatorname{Ln} \beta+h_{o} /\left(H_{O K}+H_{O R}\right)
$$

avec $\beta$ voisin de 1 .

D'après cette relation il est possible d'obtenir $K_{o}$, $\left(k_{o}\right.$ étant connu) en mesurant l'avancement de la réaction $R$ pour plusieurs quantités de catalyseur (toute autre condition étant identique) et de porter $\operatorname{Ln}[1 /(1-R)]$ en fonction de la hauteur de la phase dense (ou du catalyseur au repos). Les points doivent, si le modèle est correct, se trouver sur une droite dont la pente est égale à $H_{O K}$ pour $k_{o} \gg K_{o}$.

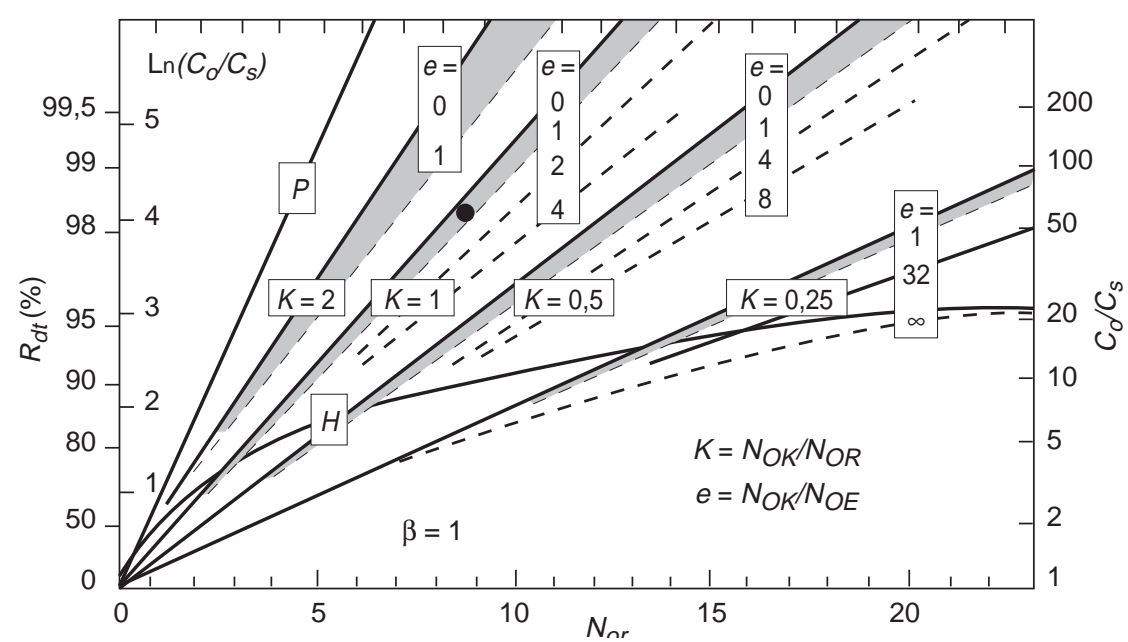

Figure 3

Représentation du modèle de May (1959) pour une réaction chimique du premier ordre proposé par Botton et al. (1970, 1980 et 1981). Le point noir est relatif à un réacteur de $: h_{o}=6 \mathrm{~m}$, avec $U-U_{o}=0,6 \mathrm{~m} / \mathrm{s}, H_{O K}=0,75 \mathrm{~m}, H_{O E} \cong 0,4 \mathrm{~m}$ et $1 / k_{o}=1,25 \mathrm{~s}$ (d'où : $N_{O R}=8, N_{O K}=8$, $N_{O E} \cong 15$ ). La courbe $H$ est relative à un réacteur mélangé idéal ( $N_{O E}=0$ et $N_{O K}$ infini) et la courbe $P$ à un réacteur piston idéal ( $N_{O E}$ et $N_{O K}$ infinis).

Representation of May's model for a first order chemical reaction proposed by Botton et al. (1970, 1980 and 1981). The black point corresponds to a plausible industrial reactor $\left(h_{o}=6 \mathrm{~m}, U-U_{o}=0.6 \mathrm{~m} / \mathrm{s}, H_{O K}=0.75 \mathrm{~m}, H_{O E} \cong 0.4 \mathrm{~m}\right.$ and $1 / \mathrm{k}_{o}=1.25 \mathrm{~s}$ from where $\left.N_{O R}=8, N_{O K}=8, N_{O E} \cong 15\right)$. $H$ is the curve of an ideal mixed flow reactor $\left(N_{O E}=0\right.$ and $N_{O K}$ very large). $P$ is the curve of an ideal plug flow reactor $\left(N_{O E}\right.$ and $N_{O K}$ very large). 


\subsubsection{En utilisant un traceur solide (méthode de May, 1959)}

L'objectif est de mesurer $E$ pour accéder à $N_{E}=U \cdot h / f_{d} E$ ou $N_{O E}=N_{E} / \beta$.

Avec un traceur solide, on mesure le coefficient de diffusion axial $D_{S}$ des particules par unité de section du lit. Dans la phase dense, la vitesse de glissement du gaz par rapport aux particules est beaucoup plus faible que celle du déplacement de la phase dense sous l'effet des bulles. Ceci permet d'admettre que dans la phase dense le gaz et les particules sont liés. En conséquence : si $D_{D}, D_{E}, D_{S}$ sont les coefficients axiaux de diffusion par unité de section de lit de la phase dense, du gaz de la phase dense et des particules, et si $\varepsilon_{o}$ est la fraction volumique du gaz dans la phase dense, on peut écrire :

$$
\begin{gathered}
D_{E}=\varepsilon_{\mathrm{o}} \cdot D_{D} \\
D_{S}=\left(1-\varepsilon_{\mathrm{o}}\right) \cdot D_{D} \\
\text { d'où : } \quad D_{E}=D_{S} \cdot \varepsilon_{\mathrm{o}} /\left(1-\varepsilon_{\mathrm{o}}\right)=f_{d} E
\end{gathered}
$$

pour $\varepsilon_{\mathrm{o}} \cong 0,5$ on obtient :

$$
D_{E} \cong D_{S} \text { (hypothèse de May) }
$$

Pour effectuer la mesure, une petite quantité de solide radioactif est répartie au sommet du lit, puis on suit sa dispersion dans le lit en fonction du temps au moyen de compteurs placés à différentes hauteurs. Les courbes obtenues convergent vers une valeur unique qui correspond au mélange homogène. Selon les courbes théoriques, si $h$ est la hauteur du lit et $t$ le temps, le mélange est homogène à $1 \%$ pour $D_{S} \cdot t / h=0,5$ et à $10 \%$ pour $D_{S} \cdot t / h=0,25$.

\subsubsection{En utilisant un traceur gaz}

Les mesures et leurs interprétations sont toujours délicates et peu précises. Le gaz traceur doit être inerte vis-à-vis du solide (non absorbable).

\section{Méthode de May (1959) et méthodes dérivées}

Le gaz traceur est injecté dans l'alimentation à la concentration $C_{o}$ (1\% environ) jusqu'à stabilisation de sa teneur $C_{S}$ dans les gaz de sortie. Lorsque cette situation est atteinte $\left(C_{o}=C_{S}\right)$ l'injection est coupée. L'évolution de $C_{S}$ est suivie en fonction du temps $t$. Pour $C_{S} / C_{o}<0,4$, les calculs et l'expérience montrent que $C_{S} / C_{o}$ évolue selon une courbe assimilable à une exponentielle. Sa constante de temps $(-S)$ est la seule grandeur retenue par May (1959), après l'avoir normée par rapport au temps de séjours moyen $\theta_{m}$ des gaz dans le lit. Les équations doivent être résolues numériquement. Elles donnent $S=f(\beta$, $\left.\varphi, N_{O K}, N_{O E}\right)$. Un exemple est donné sur la figure 5.

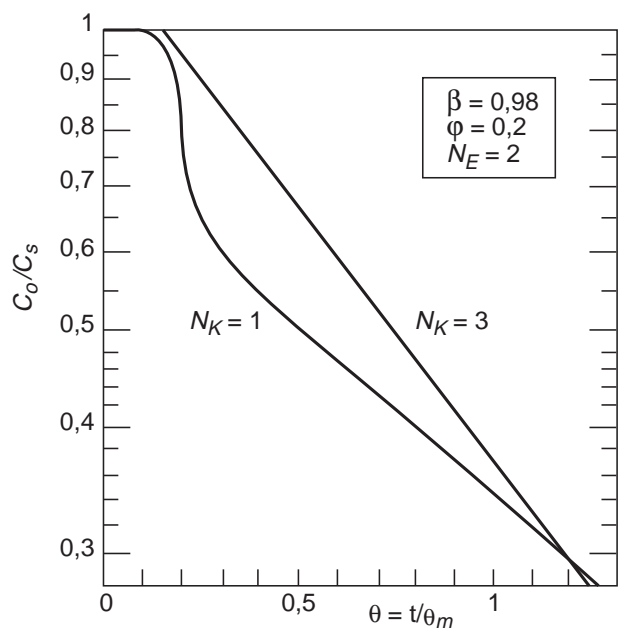

Figure 4

Exemples de courbes de réponse à une injection de type échelon. Effet de $N_{O K}=N_{K} / \beta$ pour $\beta=0,98, \varphi=0,2$ et $N_{\text {OE }}=N_{E} / \beta=2 / \beta$.

Examples of tracer output signal to a step-input signal. Effect of $N_{O K}=N_{K} / \beta$ with $\beta=0.98, \varphi=0.2$ et $N_{O E}=N_{E} / \beta=2 / \beta$.

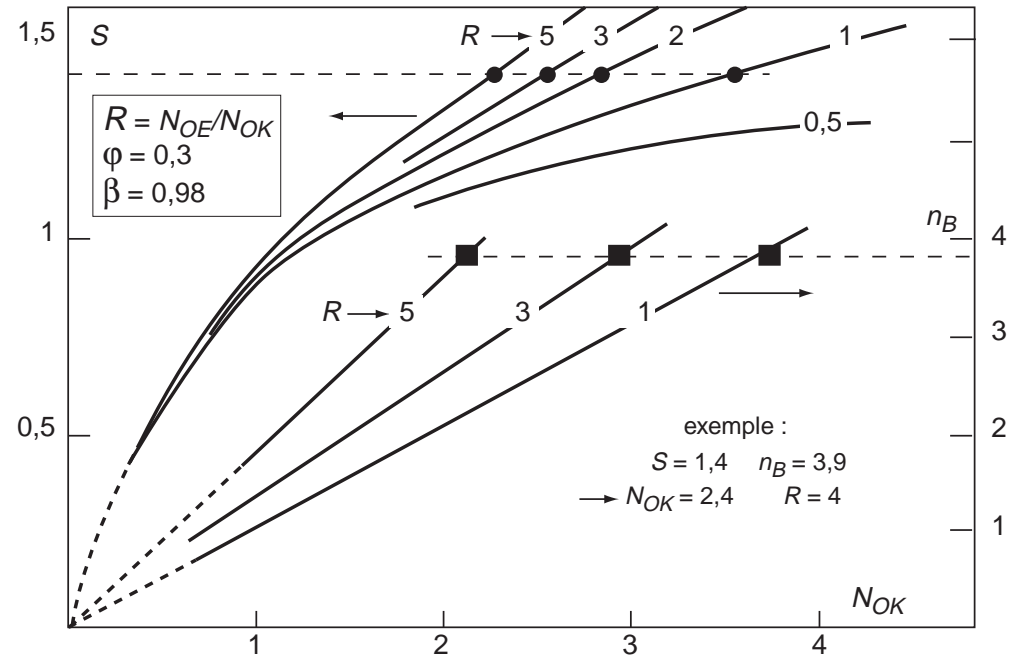

Figure 5

Exemple d'un graphique de $S$ et $n_{B}$ en fonction de $N_{K}$ et $N_{E} / N_{K}$ pour $\beta=0,98$ et $\varphi=0,3\left(n_{B}\right.$ est indépendant de $\varphi$ ) avec un exemple d'application (résultats de mesures). Pour $S=1,4$ et $n_{B}=3,9$, le tracé des deux courbes $R=f\left(N_{O K}\right)$ donne : $R=4$ et $N_{O K}=2,4$.

One graphical example of variation of $S$ and $n_{B}$ against $N_{K}$ and $N_{E} / N_{K}$ with $\beta=0.98$ and $\varphi=0.3$ $\left(n_{B}\right.$ is independant of $\varphi$ ), with application of an experimental result. For $S=1.4$ and $n_{B}=3.9$, plot of the two curves $R=f\left(N_{O K}\right)$ gives $R=4$ and $N_{O K}=2.4$. 
$N_{O K}$ est déduit des représentations graphiques de $S=f\left(\beta, \varphi, N_{O K}, N_{O E}\right)$ après avoir déterminé sur le lit $\beta, \varphi, \theta_{m}$ puis $N_{O E}$ avec un traceur solide et $S$ avec le traceur gaz.

Pour éviter l'utilisation d'un traceur solide, Botton (1970) effectue des mesures de $S$ sur deux lits de hauteur différente (toute autre condition étant inchangée). Les représentations graphiques de $S$ permettent de tracer, pour chaque valeur de $S$ mesurée, une courbe $f_{S 1}\left(N_{O K}, N_{O E}\right)=0$, puis de la transformer en courbe $g_{1}\left(N_{O K} / h_{o}, N_{O E} / h_{o}\right)=0$. Le point commun des deux courbes obtenues avec les deux valeurs de $S$ donne $N_{O K}$ et $N_{O E}$ pour un mètre de hauteur de phase dense. Dans certaines circonstances (cas où $N_{O K}$ est faible en particulier) $S$ permet d'accéder à $N_{O K}$ sans connaître $N_{O E}$. La figure 4 donne un exemple de calcul de $S$ et la figure 5 un exemple de ses variations avec $N_{O K}$ et $N_{O E}$.

\section{Méthode de Van Deemter (1961)}

Van Deemter (1961) a simplifié les équations de May en prenant $U_{o}=0$. Il a analysé en premier le cas d'une injection permanente dans le lit pour interpréter les résultats de Gilliland et Mason (1952).

Le traceur gaz est injecté en continu et uniformément dans une section transversale du lit. Si l'on échantillonne avec la même technique dans des plans situés entre le plan d'injection et le bas du lit, on trouve que la concentration en traceur décroît de façon exponentielle avec la distance $z$ au plan d'injection. Van Deemter (1961) a proposé une solution analytique des équations et montré que l'évolution de nature exponentielle observée correspondait à la relation :

$n_{B}=h \cdot d\left(\operatorname{Ln} C_{o} / C_{s}\right) / d z=\left(N_{O K} / 2\right)\left[\left(1+4 N_{O E} / N_{O K}\right)^{0,5}-1\right]$

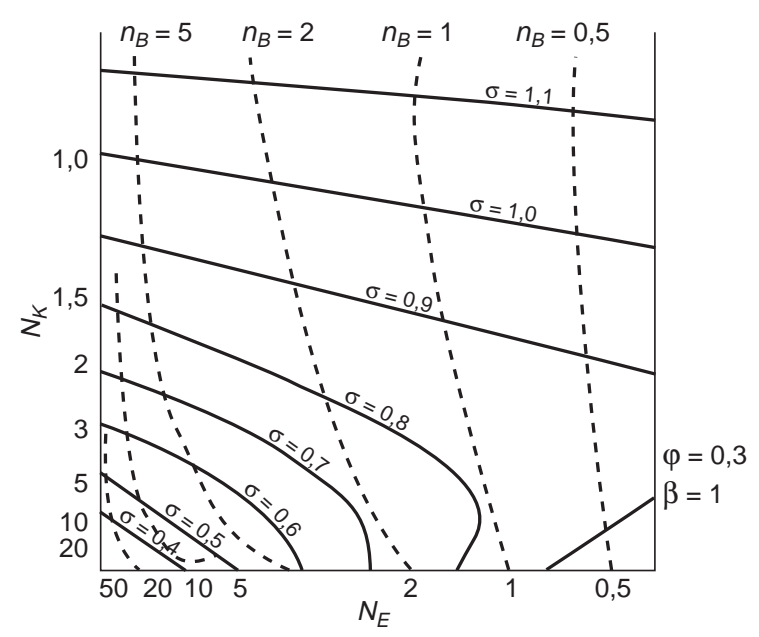

Figure 6

Exemple d'un graphique de $\sigma$ et $n_{B}$ en fonction de $N_{K}$ et $N_{E}$, établi par Van Deemter (1961).

One graphical exempla of $\sigma$ and $n_{B}$ variations against $N_{K}$ and $N_{E}$ calculated by Van Deemter (1961).
Pour disposer d'une deuxième relation entre $N_{O K}$ et $N_{O E}$, il propose de remplacer l'injection échelon de May par une injection impulsion. Les équations (avec $U_{o}=0$ ) peuvent être résolues par transformée de Laplace. Le résultat est une expression de la déviation relative standard du temps de séjour moyen du gaz traceur $\sigma=\mathrm{f}\left(N_{K}, N_{E}, \varphi\right) . n_{B}, \sigma$ et $\varphi$ étant mesurés, les graphiques de Van Deemter donnent $N_{K}$ et $N_{E}$. La figure 6 représente l'un de ces graphiques (avec $\varphi=0,3)$.

On peut de même utiliser $n_{B}$ avec $S$ (un exemple, déduit de Botton (1980) est donné figure 5).

\subsubsection{Commentaires sur les méthodes utilisant un gaz traceur}

May (1959) n'a retenu de la courbe de réponse à une injection échelon que la constante de temps qui contrôle la chute finale de la concentration en traceur. Toute une partie de la courbe de réponse est ignorée. Ceci apparaît, a priori, comme une perte d'information. En fait, l'expérience montre que cette méthode apporte simplicité et sécurité par rapport à celles qui prennent en compte la totalité du signal.

Le lit examiné, les écoulements du gaz avant et après le lit, le dispositif d'injection du gaz traceur et celui de prélèvement des échantillons en sortie ont des défauts par rapport aux hypothèses retenues pour écrire les équations. Ceci peut nuire à l'interprétation des mesures. Les expériences présentées ci-après montrent que le fait de ne retenir que l'exponentielle finale atténue ces imperfections. Au préalable, rappelons que si une injection échelon est remplacée par une injection impulsion, la courbe de réponse obtenue est une dérivée de la première. Donc, les exponentielles qui gèrent les décroissances finales des deux courbes ont la même constante de temps.

Les expériences ont été faites dans un appareil de 0,25 m de diamètre et de $3 \mathrm{~m}$ de hauteur, dont $1 \mathrm{~m}$ était occupé par un lit de particules fines (60 microns) fluidisées à la vitesse de $0,2 \mathrm{~m} / \mathrm{s}$. L'enregistrement du logarithme de la concentration en gaz traceur de l'échantillon prélevé en sortie a permis de faire les constatations suivantes :

- La forme du signal d'entrée (même la plus fantaisiste) n'a pas eu d'incidence sur la constante de temps de la dernière exponentielle de décroissance obtenue après coupure définitive de l'injection. Ceci s'explique si les injections sont assimilées à des séries d'injections impulsion qui donnent des exponentielles de constante de temps identiques et dont la somme est une exponentielle de même constante de temps.

- La valeur $S$ de cette constante de temps s'est montrée également indépendante de la position de la sonde au-dessus du lit (déjà constaté par Botton (1970) avec $D_{T}=0,5$ et $\left.1,8 \mathrm{~m}\right)$.

- La sonde a été placée au sommet de l'appareil, puis coiffée avec des capacités cylindriques de même diamètre et de 
différentes longueurs percées de trous disposés pour bien mélanger le gaz prélevé (12 1/min) à celui déjà présent dans la capacité. Des déformations importantes apparaissent dans la première partie de la courbe de réponse, mais la valeur de $S$ reste inchangée si le rapport entre le temps de séjour moyen du gaz dans la capacité qui coiffe la sonde et son temps de passage dans la zone fluidisée est inférieur à 0,5. En revanche, si ce rapport atteint 2, l'effet est inacceptable.

- Les auteurs qui ont utilisé une injection impulsion suivie du calcul de la déviation relative standard (qui concerne toute la courbe de réponse) ont été obligés, pour interpréter leurs résultats, d'installer un agitateur au-dessus du lit, et de considérer que la courbe de réponse était celle du lit fluidisé et d'un réacteur mélangé branchés en série.

La figure 4 montre que la courbe de réponse à une injection de type échelon est assimilable à deux demi-droites de pentes 0 et $-S$ pour $N_{K}>3$. Dans ces conditions le calcul de sa déviation relative standard $s$ conduit à la relation $\sigma=(1 / S)$.

\subsection{Exemples de travaux expérimentaux}

\subsubsection{Introduction}

L'objectif est de déterminer comment $K_{o}$ et $E_{o}$ évoluent en fonction des conditions opératoires en vue de dégager les tendances à suivre pour avoir des réacteurs performants. Trois sociétés au moins ont utilisé cette démarche et publié une partie de leurs résultats : Esso (avec laquelle May travaillait), Shell (procédé Deacon) et Péchiney - Saint-Gobain (PSG) (oxychloration de l'éthylène en non sélectif). Notons que les phases actives des catalyseurs utilisés dans ces deux derniers procédés sont fluides, ce qui accentue les difficultés. C'est à partir des travaux de ces trois sociétés que nous articulerons notre présentation.

Nous examinerons les facteurs d'action prospectés, indiquerons les granulométries des poudres étudiées, aborderons le problème de la qualité d'une fluidisation, exposerons les mesures relatives à $K_{o}$ et $E_{o}$. Enfin, nous terminerons en dégageant les tendances à suivre pour obtenir des catalyseurs performants en contact gaz-solide. Ces tendances ont été en partie quantifiées avec des graphiques de synthèse.

Un aperçu de la dimension des équipes qui ont été nécessaires dans les trois sociétés nommées auparavant est donné par la liste des auteurs des publications et des participants cités en remerciements :

- Shell : Van Deemter, De Groot, Van Swaaij, Zuiderweg, De Vries, Mantovani, Heijkoop, Bohle ;

- PSG : Botton (extrapolation), Delcoigne (maquettes et traceurs), Rouault, Henin, Corbin (calcul), Kaziz, Antonini, (chimie) Lainé, Joffre, (pilotes) ;

- Esso : May, Schulman (théorie), et plus ou moins, d'après leurs travaux : Lewis, Murphree, Gilliland, Mason, Matheson, Matsen, etc.

\subsubsection{Paramètres d'action à examiner}

Ces différents paramètres sont :

- Pour la poudre : le diamètre moyen des particules, leur granulométrie, leur masse spécifique apparente, leur porosité, leur état de surface (rugosité, tendance à l'agglomération), leur fragilité.

- Pour les conditions de travail : la vitesse des gaz, leur nature, la température, la pression, la nature du traitement effectué (chimique catalytique ou non, physique).

- Pour la géométrie des appareils : le diamètre, la hauteur, le distributeur du gaz à l'entrée du lit, les échangeurs de chaleur, le captage et le recyclage des particules entraînées.

\subsubsection{Choix des poudres}

Murphree (1949) a indiqué à l'issue des travaux de mise au point des premiers ateliers de craquage catalytique, qu'un mélange de particules de 10 à 150 microns avec un diamètre moyen de 62 microns donne une excellente fluidisation.

Matheson et al. (1949) ont mis en évidence que la viscosité et la qualité d'une fluidisation sont liées. Les grosses particules donnent des lits plus visqueux que les fines.

- Si des particules fines sont ajoutées à des grosses particules, la viscosité décroît très vite puis très lentement. (Matheson et al. attribuent à ceci le fait que le comportement d'un lit industriel peut se montrer très sensible à l'addition (ou la perte) de petites quantités de particules fines.

- Au point de transition, la quantité de particules fines ajoutée est proche de celle nécessaire à enrober les grosses particules. Cette quantité, fonction des tailles des particules, a été formulée par Tarmy selon Zenz et Othmer (1960).

- Zenz et Othmer (1960) se sont servis de cette formulation pour calculer la répartition granulométrique la plus favorable pour une poudre constituée de 10 fractions de particules dont les diamètres extrêmes sont dans un rapport de 10 . La répartition trouvée est voisine de celle des catalyseurs de craquage catalytiques. Selon Zenz et Othmer, elle correspond à ce que l'on obtient par broyage. Ce traitement a tendance à optimiser la fluidité d'une poudre.

- Représentée sur un diagramme de Rosin-Ramler (log $\cdot \log$ $(100 /$ refus cumulé en $\%)$ en fonction de $\log \cdot d p)$ cette répartition donne une droite selon laquelle le rapport des ouvertures des tamis qui retiennent 10 et $50 \%$ de la poudre (en poids) est de 1,6 environ $\left(d_{10} / d_{50}=1,6\right)$.

La figure 7 représente un diagramme de Rosin-Ramler sur lequel nous avons reporté la répartition granulométrique calculée par Zenz et Othmer (celle des catalyseurs de craquage catalytiques) et celles utilisées dans les travaux pris en compte dans cette étude.

- May (1959) a utilisé un support de catalyseur de craquage catalytique (FCC) qui avait perdu ses particules fines.

- De Groot (1967) a choisi deux sables broyés de 150 et $210 \mu \mathrm{m}$ de $d_{50}$ et dont les répartitions granulométriques étaient très dispersées pour le premier et très étroites pour l'autre. 


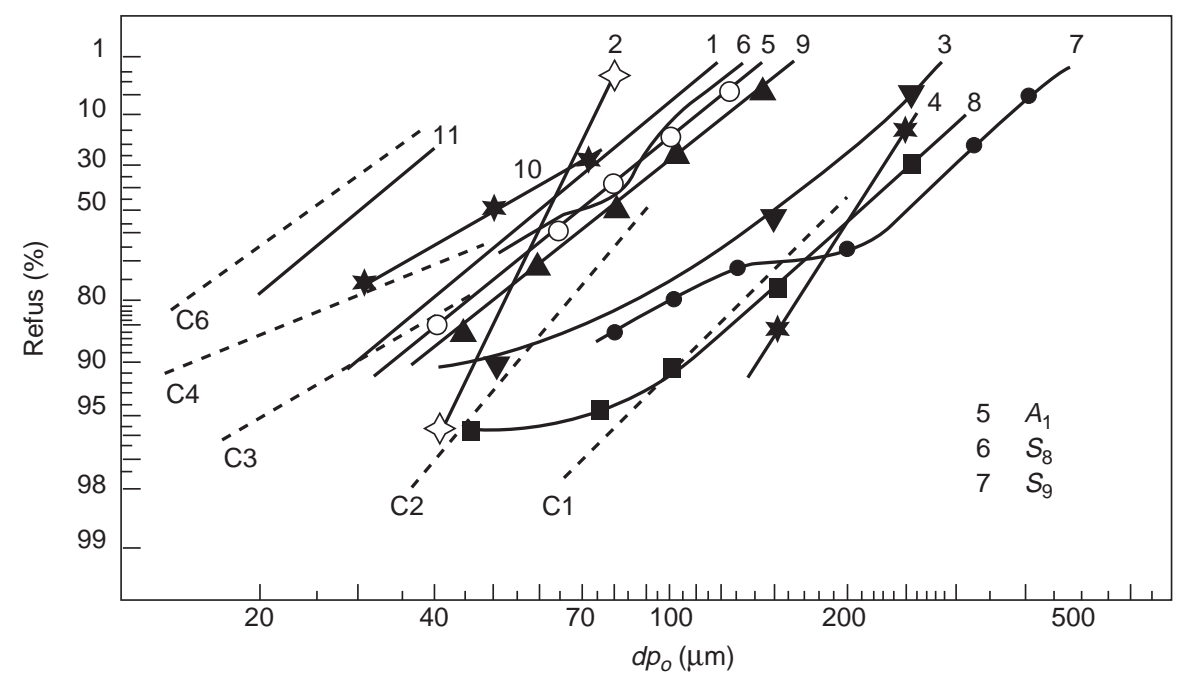

Figure 7

Répartition granulométrique des poudres étudiées :

Granulometric dispersion of studied powders:

1 : courbe théorique de Zenz et Othmer (1960), catalyseur de craquage ;

$2:$ support de catalyseur de craquage utilisé par May (1959);

3 et 4 : sables à forte et faible dispersion granulométrique de De Groot (1967) ;

5,6 et 7 : support de catalyseur de craquage $\left(A_{1}\right)$ et sables $\left(S_{8}\right.$ et $S_{9}$ ) utilisés par Botton (1970) ;

8 et 9 : sable et silice atomisée utilisés par Van Swaaij (1972);

9, 10 et 11 : silices atomisées et grade A de De Vries (1972);

C1 à C6 : cokes n 1, 2, 3, 4 et 6 utilisés par Matsen (1970).

1: theoretical Zenz and Othmer's curve and Cracking

Catalyst;

2: cracking catalyst carrier used by May (1959);

3 and 4: sands with large and narrow granulometric dispersion used by De Groot (1967);

5, 6 and 7: cracking catalyst carrier $\left(A_{1}\right)$ and sands $\left(S_{8}\right.$ and $S_{9}$ ) used by Botton (1970);

8 and 9: sand and sprayed silica used by Van Swaaij (1972);

9, 10 and 11: sprayed silica and grad A used by de Vries (1972);

C1 to C6: cokes no. 1, 2, 3, 4, and 6 used by Matsen (1970).

- Botton (1970) a utilisé, en adoptant au mieux la répartition théorique de Zenz et Othmer, un support de catalyseur de craquage catalytique $\left(A_{1}, d_{50}=70 \mu \mathrm{m}\right)$, deux sables $\left(S_{8}\right.$ et $S_{9}$ de $d_{50}=70$ et $250 \mu \mathrm{m}$ ) et des catalyseurs d'oxychloration d'éthylène de $d_{50}>150 \mu \mathrm{m}$. La différence principale entre $A_{1}$ et $S_{8}$ est la densité apparente de leurs particules $(0,75$ et 2,5$)$.

- De Vries et al. (1972) ont utilisé de la silice atomisée $\left(d_{50}=80 \mu \mathrm{m}\right)$ dont ils ont fait varier la quantité de particules $<44$ microns et un catalyseur d'oxydation d'acide chlorhydrique en chlore.

- Van Swaaij et Zuiderweg (1972) ont utilisé un sable $(200 \mu \mathrm{m})$ et une silice atomisée $\left(d_{50}=80 \mu \mathrm{m}\right)$.

\subsubsection{Qualité d'une fluidisation, le phénomène bulles}

\section{Les effets de la granulométrie sont importants}

Matsen (1973) en a donné une description très détaillée à l'aide de six poudres, préparées avec du coke de pétrole (de densité 2). Elles ont été fluidisées avec de l'air à des vitesses de 0,1 à $0,4 \mathrm{~m} / \mathrm{s}$. L'appareil en Plexiglas, de $0,6 \mathrm{~m}$ de diamètre et de 3,6 m de hauteur, était muni à sa base d'un simple cône pour distribuer le gaz et, le long d'une génératrice, de prises de pression tous les $0,15 \mathrm{~m}$. La figure 8 donne un schéma de l'appareil et les granulométries des poudres.
Celles-ci ont été reportées en partie sur la figure 7.

- Le coke $\mathrm{n}^{\circ} 1\left(d_{50}=200 \mu \mathrm{m}, 0 \%<40 \mu \mathrm{m}\right)$ et le coke $\mathrm{n}^{\circ} 2$ $\left(d_{50}=90 \mu \mathrm{m}, 1 \%<40 \mu \mathrm{m}\right)$ ont donné de très grosses bulles accompagnées de violentes vibrations.

- Le coke $\mathrm{n}^{\circ} 3\left(d_{50}=80 \mu \mathrm{m}, 18 \%<40 \mu \mathrm{m}\right)$ a donné une fluidisation douce, avec une montée des bulles au centre, une descente de la poudre le long des parois (circulation Gulf Stream). À un niveau donné, les pressions diminuent lorsqu'on se déplace des parois vers le centre du lit.

- Avec le coke $\mathrm{n}^{\circ} 4\left(d_{50}=70 \mu \mathrm{m}, 30 \%<40 \mu \mathrm{m}\right)$ les bulles sont uniformément réparties dans le lit et présentes aux parois. Elles sont issues de la fragmentation instantanée de bulles de 0,25 $\mathrm{m}$ de diamètre qui émergent du cône distributeur. Le solide ne descend plus de préférence le long des parois. Les pressions sont constantes sur toute la section à un niveau donné. Toutes les vibrations ont disparu.

- Le coke $\mathrm{n}^{\circ} 6\left(d_{50}=25 \mu \mathrm{m}, 85 \%<40 \mu \mathrm{m}\right)$ obtenu par élutriation des particules fines des cokes précédents a été essayé dans un appareil de $0,11 \mathrm{~m}$ de diamètre. Les observations ont été identiques à celles effectuées avec le coke $\mathrm{n}^{\circ} 4$, mais avec des bulles plus petites $(25 \mathrm{~mm})$. 


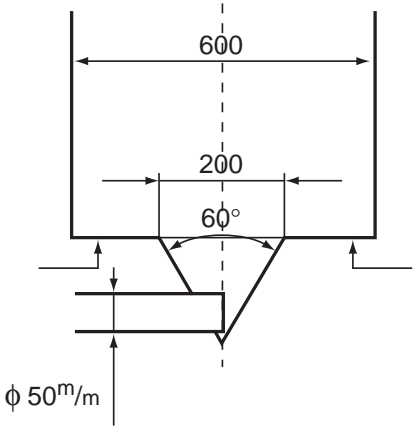

Distributeur 1 cône

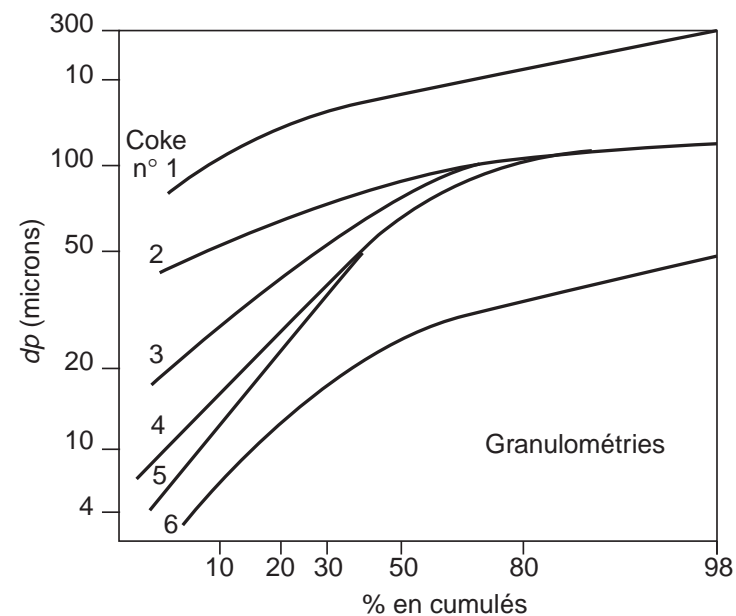

Figure 8

Appareil et granulométrie des poudres utilisées (cokes de pétrole) par Matsen (1970) pour étudier le phénomène bulles. Device and granulometry of powder (petroleum cokes) used by Matsen (1970) to study bubble phenomena.

\section{Les forces d'agglomération peuvent jouer un rôle décisif}

De Vries et al. (1972) ont obtenu, en chauffant un lit fluidisé de particules très fines $\left(d_{50} \cong 30 \mu \mathrm{m}, \cong 20 \%<20 \mu \mathrm{m}, \mathrm{n}^{\circ} 11\right.$ sur la figure 7) à $300^{\circ} \mathrm{C}$, une structure moussante (très petites bulles et très grande expansion). Ils ont montré que l'apparition de cet état correspondait à la disparition de forces d'agglomération entre les particules dues dans ce cas à des produits adsorbés.

\section{La densité des particules a un effet important}

La figure 9 de Botton (1970) donne la dimension des bulles observées au sommet d'un lit de $0,5 \mathrm{~m}$ de diamètre et de $3,5 \mathrm{~m}$ de hauteur pour les poudres $S_{8}$ et $A_{1}$ de granulométrie voisines $\left(d_{50} \cong 70 \mu \mathrm{m}\right.$, fig. 7$)$ mais de densités de grain très différentes $\left(d a_{p}=2,5\right.$ et 0,75$)$.

- Avec $S_{8}\left(d a_{p}=2,5\right)$ le diamètre des bulles augmente avec la vitesse des gaz $U$, puis se stabilise vers $0,3 \mathrm{~m}$ pour $U>0,2 \mathrm{~m} / \mathrm{s}$. La vitesse d'augmentation du diamètre est ralentie et sa valeur limite abaissée lorsqu'on immerge des tubes verticaux simulant un échangeur industriel.

- Avec $A_{1}\left(d a_{p}=0,75\right)$, l'aspect des bulles est resté le même dans toutes les conditions. Leur diamètre était de l'ordre de 3 à $5 \mathrm{~cm}$.

Les tubes verticaux d'un échangeur ne limitent pas le diamètre des bulles à l'espace libre entre tubes

Les bulles générées par du sable voisin de $S_{9}\left(d_{50} \cong 250 \mu \mathrm{m}\right.$, fig. 7) ont été observées par Botton (1970) dans un lit de $1,8 \mathrm{~m}$ de diamètre à une vitesse de gaz de $0,2 \mathrm{~m} / \mathrm{s}$. Des tubes verticaux étaient immergés dans le lit. Leur diamètre était de $\phi=89 \mathrm{~mm}$. Ils étaient disposés en mailles carrées de $2 \phi$. À $1 \mathrm{~m}$ du distributeur, les bulles cheminaient entre les tubes, à $2 \mathrm{~m}$ leur taille leur permettait d'enrober chacune un tube. À $3 \mathrm{~m}$, chacune enrobait quatre tubes.

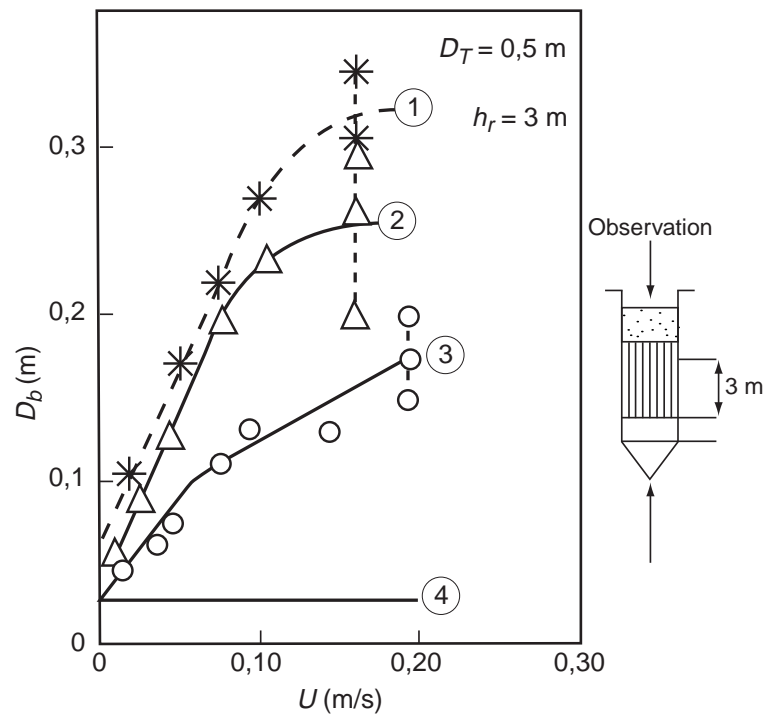

Figure 9

Sensibilité de la dimension des bulles observées à la surface d'un lit aux caractéristiques des poudres fluidisées et à l'immersion de tubes verticaux pour un lit de $3 \mathrm{~m}$ de hauteur au repos et avec $D_{T}=0,5 \mathrm{~m}$, selon Botton (1970).

Avec le sable $S_{8},\left(d_{50}=70 \mu \mathrm{m}, d a_{p}=2,5\right.$; voir fig. $\left.7, \mathrm{n}^{\circ} 6\right)$ : 1 : fût vide $; 2: 5$ tubes de $\phi=89 \mathrm{~mm} ; 3: 12$ tubes de $\phi=60 \mathrm{~mm}$. Avec le support $A_{1}\left(d_{50} \cong 70 \mu \mathrm{m}, d a_{p}=0,75\right.$; voir fig. $\left.7, \mathrm{n}^{\circ} 5\right)$ : 4 : dans tous les cas (avec ou sans tube).

Variation of the bubble's diameters at the top of a bed against gas superficial velocity, characteristics of fluidized powders and vertical tubes immersed, with a bed of $3 \mathrm{~m}$ high when it is settled and of $0.5 \mathrm{~m}$ diameter.

With sand $S_{8}\left(d_{50}=70 \mathrm{~mm}, d a_{p}=2.5\right.$, see Fig. 7 no. 6):

1: no tube; 2: 5 tubes $\phi=89 \mathrm{~mm} ; 3: 12$ tubes $\phi=60 \mathrm{~mm}$.

With $A_{1}\left(d_{50} \cong 70 \mathrm{~mm}, d a_{p}=0.75\right.$, see Fig. 7 no. 5):

4: all conditions (with and without tube). 


\subsubsection{L'expansion des lits}

On appelle expansion l'évolution de $h$ en fonction de $U$. Pour $U$ très supérieur à $U_{m f}$ le niveau du lit fluctue sous l'effet de l'éclatement des bulles. C'est la valeur moyenne qui est retenue. Avec des particules fines, le niveau n'est plus visible aux hautes vitesses de gaz en raison des entraînements de particules. Dans cette situation, il est localisé par des relevés de pressions le long d'une génératrice.

La figure 10 donne les courbes d'expansion obtenues par Botton (1970) avec $S_{8}\left(d_{50} \cong 70 \mu \mathrm{m}\right)$ et $S_{9}\left(d_{50}=250 \mu \mathrm{m}\right)$. Avec $S_{8}$, on note un régime d'expansion rapide entre $U_{m f}$ et environ $5 U_{m f}$ suivis d'un régime lent. Des bulles apparaissent nettement au voisinage du changement de régime. Pour $S_{9}$, seul le régime lent est noté. Les bulles apparaissent à $U_{m f}$. Ces courbes d'expansion sont représentables par la relation suivante dans laquelle $U_{B X}$ est constant pour une poudre donnée :

$$
\left(h-h_{o}\right) /\left(U-U_{o}\right)=h_{o} / U_{B X}
$$

Dans cette relation, $h_{o}$ et $U_{o}$ sont la hauteur du lit et la vitesse des gaz au point de changement de régime. Pour les poudres telles que $S_{9}$ qui ne présentent que le régime d'expansion lent, on a : $h_{o}=h_{m f}$ et $U_{o}=U_{m f}$.

- Botton (1972) a admis que $h_{o}$ et $U_{o}$ représentaient la hauteur de phase dense et le débit de gaz qui la traverse, quelle que soit la valeur de $U$ (conforme au modèle de May). May avait pris pour ces valeurs celles qui correspondent à l'apparition des premières bulles.
- De Vries et al. (1972) ont mesuré les valeurs de $h_{o}$ et $U_{o}$ relatives à une valeur donnée de $U$, en utilisant la courbe d'évolution de la hauteur du lit après avoir coupé l'alimentation en gaz. Ils ont noté que $h_{o}$ et $U_{o}$ sont bien indépendants de $U$.

- $\left(h-h_{o}\right) /\left(U-U_{o}\right)$ est égal au temps de séjour des bulles dans le lit. De ce fait, le coefficient d'expansion $U_{B X}$ est égal à leur vitesse de glissement par rapport à la phase dense. Il pourrait être fonction de leur diamètre. En fait, il en est indépendant car il ne varie pas avec la hauteur du lit lorsque le diamètre des bulles augmente de bas en haut (cas des particules grossières).

La figure 11 regroupe en fonction du diamètre de l'appareil les valeurs de $U_{B X}$ déduites de divers travaux. Elle peut se commenter comme suit :

- Pour les poudres à particules fines telles que $A_{1}$ et $S_{8}$ $\left(d_{50} \cong 70 \mu \mathrm{m}\right) U_{B X}$ augmente avec le diamètre $D_{T}$ de l'appareil, puis se stabilise pour $D_{T}>0,25 \mathrm{~m}$ à $1,1 \mathrm{~m} / \mathrm{s}$ pour $S_{8}\left(d a_{p}=2,5\right)$ et à $0,6 \mathrm{~m} / \mathrm{s}$ pour $A_{1}\left(d a_{p}=0,75\right)$. L'immersion de tubes verticaux de dimensions industrielles $(\phi=46$ et 89 mm en mailles carrées de $2 \phi$ ) dans $D_{T}=0,50 \mathrm{~m}$ n'en modifie pas la valeur, alors qu'avec des tubes plus petits $(\phi=$ $17 \mathrm{~mm}$ ) dans $D_{T}=0,25 \mathrm{~m}$, elle diminue $\left(S_{8}\right)$.

- Pour les poudres à grosses particules telles que $S_{9}$ $\left(d_{50} \cong 250 \mu \mathrm{m}\right) U_{B X}$ augmente de 0,4 à $3,5 \mathrm{~m} / \mathrm{s}$ lorsque $D_{T}$ augmente de 0,1 à $1,8 \mathrm{~m}$. L'immersion de tubes verticaux $(\phi=89 \mathrm{~mm}$ en mailles carrées de $2 \phi)$ augmente $U_{B X}$ pour $D_{T}<1 \mathrm{~m}$ et le diminue pour $D_{T}=1,8 \mathrm{~m}$.

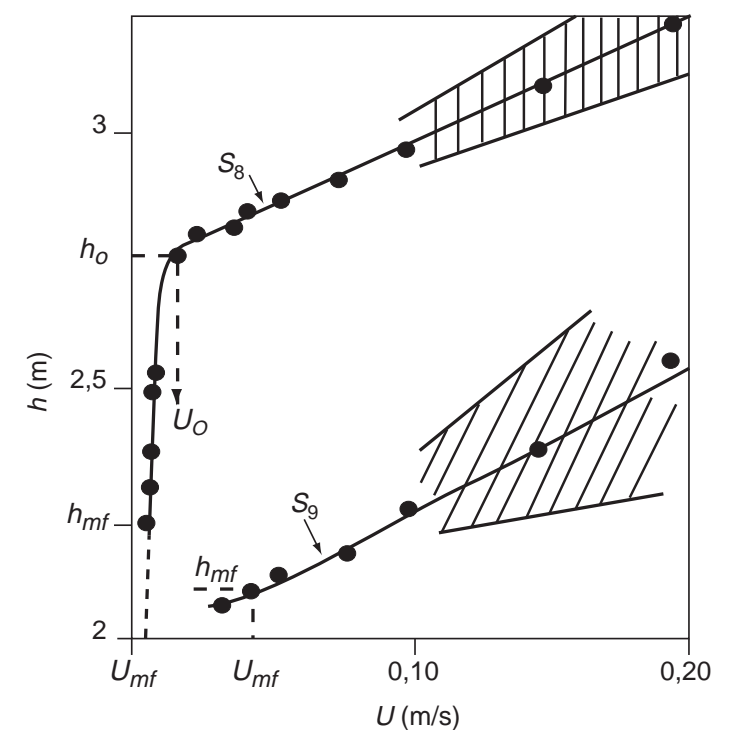

Figure 10

Hauteur d'un lit fluidisé en fonction de la vitesse des gaz avec des poudres à particules fines $\left(S_{8}: d_{50} \cong 70 \mu \mathrm{m}\right.$, voir fig. $\left.7, \mathrm{n}^{\circ} 6\right)$ et grossières $\left(S_{9}: d_{50} \cong 250 \mu \mathrm{m}\right.$, voir fig. $7, \mathrm{n}^{\circ} 7$ ), selon Botton (1970).

High of fluidized beds of fine and coarse particles $\left(S_{8}: d_{50} \cong 70 \mu \mathrm{m}\right.$ and $S_{9}: d_{50} \cong 250 \mu \mathrm{m}$ see Fig. 7 no. 6 et 7) from Botton (1970).

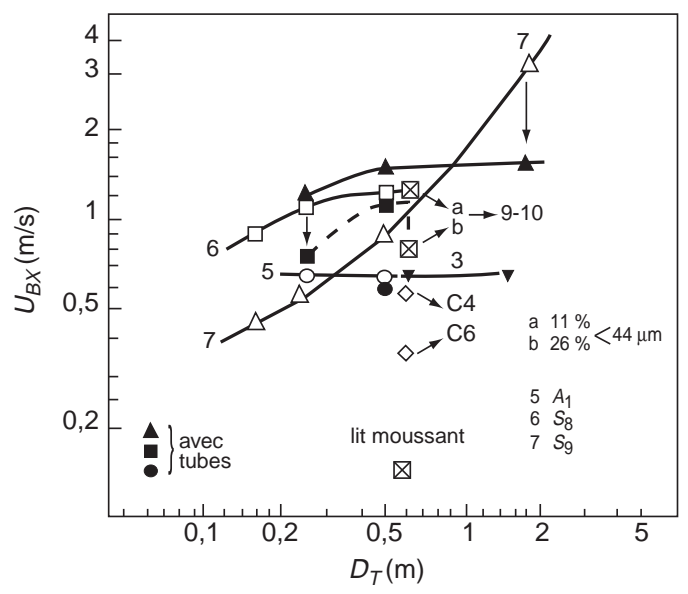

Figure 11

Coefficient d'expansion $U_{B X}$ d'un lit fluidisé en fonction du diamètre de l'appareil, de la présence ou non de tubes verticaux et de la granulométrie de la poudre. Les numéros de repères sont identiques à ceux de la fig. 7 (granulométries et auteurs). Pour les particules fines, $h_{o} / h_{m f}$ est resté inférieur à 1,20.

Expansion coefficient $U_{B X}$ of a fluidized bed against bed diameter, powders and vertical tubes. For numbers (references) see Fig. 7 (granulometry and authors). With fine particles, $h_{o} h_{m f}$ was $<1.20$. 
- Comparés à ces résultats, le sable à granulométrie étalée $\left(d_{50} \cong 150 \mu \mathrm{m}\right)$ de De Groot (1967) se comporte comme les poudres à particules fines et celui à granulométrie étroite $\left(d_{50} \cong 210 \mu \mathrm{m}\right)$ comme les poudres à grosses particules.

- L'augmentation de la teneur en particules fines de $d_{50}<40 \mu \mathrm{m}$ diminue $U_{B X}$, selon les essais de De Vries et al. (1972) et de Matsen (1970).

- On notera que les poudres qui donnent une excellente fluidisation (bulles de même taille, petites, bien réparties dans tout le lit) telles que $A_{1}$, cokes $\mathrm{n}^{\circ} 4$ et 6 ont des valeurs de $U_{B X}$ inférieures à $0,7 \mathrm{~m} / \mathrm{s}$.

\subsubsection{Mesure de $K_{\mathrm{o}}\left(H_{O K}\right)$ en utilisant une réaction chimique}

Rappelons la relation qui donne l'avancement d'une réaction :

$$
C_{S} / C_{o}=(1-R)=\beta \cdot \exp \left[-h_{o} /\left(H_{O K}+H_{O R}\right)\right]
$$

Les travaux présentés par Botton (1970) sont relatifs à une réaction d'oxychloration d'éthylène non sélective dans des réacteurs de $0,065,0,16,0,25$ et $0,5 \mathrm{~m}$ de diamètre avec des catalyseurs de $150<d_{50}<600 \mu \mathrm{m}$ et de $1 / k_{o}=0,4 \mathrm{~s}$. Les vitesses des réactifs dans la phase bulles $\left(U-U_{o}\right)$ ont évolué de 0,05 à $0,40 \mathrm{~m} / \mathrm{s}$ et la pression de 1 à 5 bar.

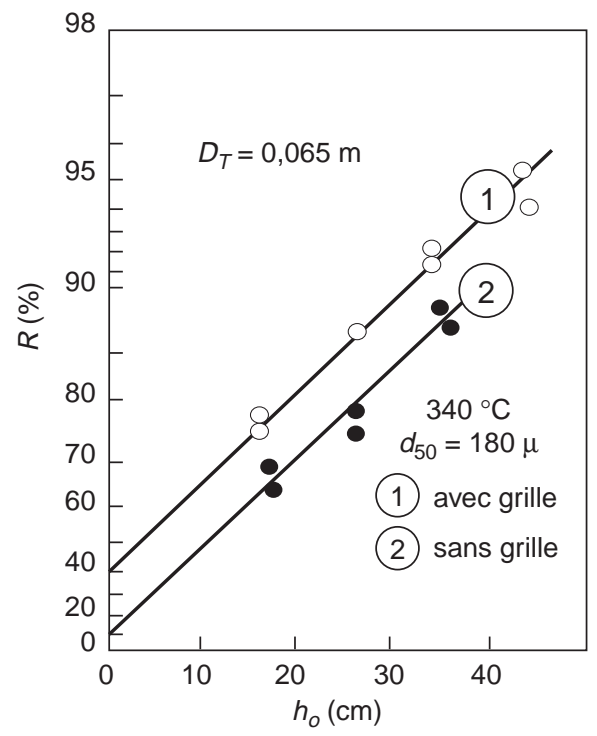

Figure 12a

Représentation de l'avancement d'une réaction chimique en fonction de la hauteur de catalyseur avec $D_{T}=0,065 \mathrm{~m}$, suivant le modèle de May. Mise en évidence de "l'effet de grille" (Botton, 1970).

Plot of the yield of a $D_{T}=0.065 \mathrm{~m}$ chemical reactor against high of the catalyst settled bed as suggests May'model. It is evident that gas distributor as an effect ("grid effect"). From Botton (1970).

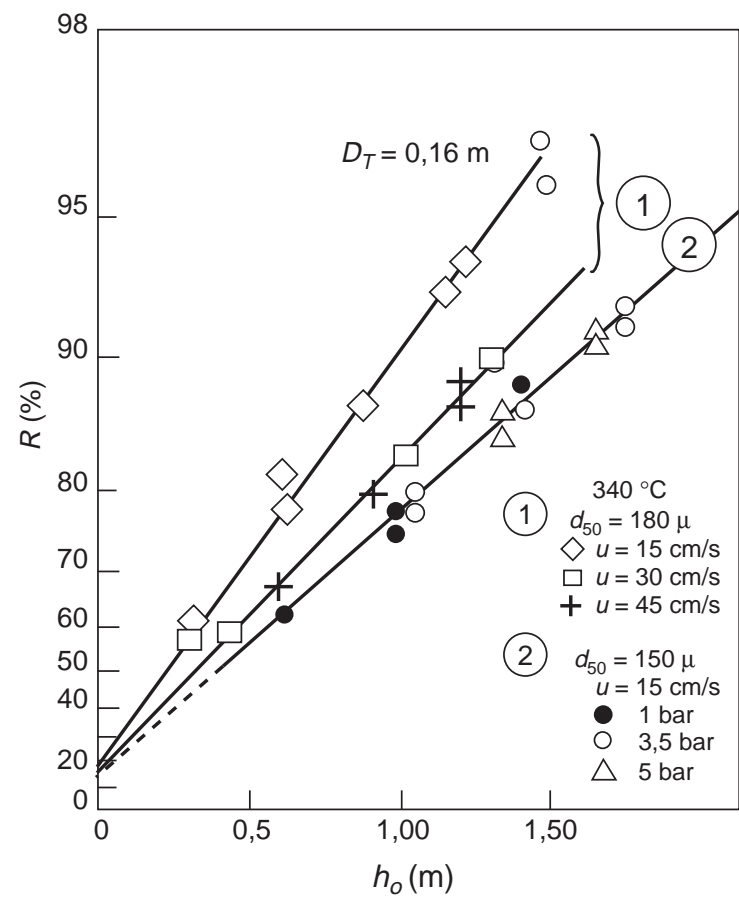

Figure 12b

Identique à la figure $12 \mathrm{a}$ : effet, avec $D_{T}=0,16 \mathrm{~m}$ de la vitesse des gaz, de la pression et de la granulométrie selon Botton (1970). Identic to Figure $12 a$ with $D_{T}=0.16 \mathrm{~m}$. Variation of the yield against superficial velocity, pressure and granulometry (Botton 1970).

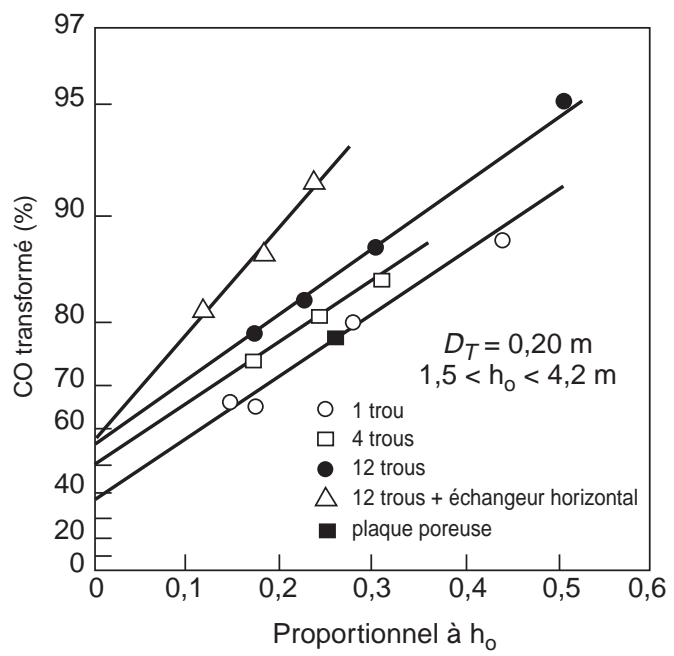

Figure 12c

Interprétation des expériences de Greckel (1964) par Botton (1970). Effet du nombre d'orifices d'émissions à débit constant de réactif pour une section totale des orifices de 0,042 fois celle du lit de diamètre $D_{T} \cong 0,20 \mathrm{~m}$. Confirmation du phénomène "effet de grille".

Results of Greckel's experiments (1964) plotted by Botton (1970). Superficial velocity of gas was constant and grids used had the same free area $(0.042$ of the reactor section of $0.20 \mathrm{~m}$ diameter) "grid effect" is yet evident. 
- Les résultats ont été examinés en portant sur un graphique : $[-\log (1-R)]=\log \left(C_{o} / C_{S}\right)$ en fonction de $h_{o}$, avec en réseau le paramètre étudié. Les figures $12 \mathrm{a}$ et $12 \mathrm{~b}$ en sont deux exemples.

- Les courbes obtenues sont des droites, ainsi que le prévoit le modèle, mais elles ne passent pas par l'origine. Deux causes sont possibles :

- selon la relation (5) pour $h_{o}=0:-\log (1-R)=-\log \beta$. C'est l'effet de la fraction de réactifs qui s'écoule par la phase dense ;

- la zone voisine de la grille où se forment les bulles et celle du haut du lit où elles éclatent sont deux zones privilégiées pour le contact gaz-solide. Cet effet est, pour le domaine étudié, supérieur au précédent. Il a été appelé "effet de grille".

- L'effet de grille est mis en évidence par la figure 12a (essai avec et sans grille). La droite obtenue "sans grille" est parallèle à celle "avec grille" et passe par l'origine. Ceci est illustré en détail par les essais de Greckel (1964) lorsqu'on les porte (fig. 12c) dans le même système de coordonnées. Ces essais donnent une série de droites parallèles. Leurs ordonnées à $h_{o}=0$, représentent les "effets de grille". Ceux-ci augmentent avec le nombre de trous de la grille à taux de perforation constant (4\%). On y note aussi que les plaques poreuses ne sont pas efficaces. L'immersion d'un échangeur améliore le contact gaz-solide dans le lit, sans modifier celui de la zone proche de la grille. L'effet de grille est le même.

- Selon la figure $12 b$, les pentes des droites obtenues sont indépendantes de la pression (de 1 à 5 bar absolus) et évoluent avec $\left(U-U_{o}\right)$ pour en devenir indépendantes. Les valeurs correspondantes de $K_{o}$ sont indépendantes puis proportionnelles à $\left(U-U_{o}\right)$ (donc $H_{O K}$ proportionnel puis indépendant de $\left.U-U_{o}\right)$. Les vitesses $\left(U-U_{o}\right)$ auxquelles s'effectue la transition ont été de $0,11,0,16$ et $0,22 \mathrm{~m} / \mathrm{s}$ pour les réacteurs de $0,065,0,16$ et $0,50 \mathrm{~m}$ de diamètre (celui de 0,5 ayant un échangeur immergé). Ces valeurs correspondent à :

$$
U-U_{o} \cong 0.12\left(g D_{T}\right)^{0,5}
$$

- Les valeurs de $1 / H_{O K}$ indépendantes de $\left(U-U_{o}\right)$ ont été portées sur la figure 13 en fonction de $U_{o}$ pour plusieurs réacteurs. $1 / H_{O K}$ augmente quand $U_{o}$ augmente. L'effet s'atténue quand le diamètre $D_{T}$ du réacteur augmente et s'inverse pour $D_{T}>0,25 \mathrm{~m}$ d'après quelques tests faits dans un réacteur de $D_{T}=0,25 \mathrm{~m}$ (sans échangeur de chaleur). $\mathrm{Si}$ l'effet subsiste un peu sur le réacteur pilote de $D_{T}=0,5 \mathrm{~m}$, c'est en raison de la présence d'un échangeur de chaleur (obligatoire pour cette réaction). Il retarde le grossissement des bulles. Pris à l'état brut, les résultats présentés sur la figure 13 incitent (en l'absence du test avec $D_{T}=0,25 \mathrm{~m}$ ) à utiliser des grosses particules. C'est un piège redoutable, car utiliser des grosses particules dans des grands lits c'est favoriser l'existence de zones défluidisées, les faibles coefficients de transfert de chaleur ainsi que les phénomènes de vibration et d'érosion. C'est l'échec garanti.

- Les valeurs de $H_{O K}$, obtenues en extrapolant $U_{o}$ à $U_{o}=0$ sur la figure 13 , ont été retenues comme des valeurs très accessibles avec des particules fines. Elles sont de 0,33, 1 et $2 \mathrm{~m}$ pour $D_{T}=0,065,0,16$ et $0,50 \mathrm{~m}$. Elles ont été portées sur la figure 14 .

De Vries et al. (1972) ont étudié l'incidence de la teneur en particules fines du catalyseur du Shell Chlorine Process sur l'avancement de la réaction dans un réacteur de $3 \mathrm{~m}$ de diamètre et de $10 \mathrm{~m}$ de lit fluidisé à $U=0,2 \mathrm{~m} / \mathrm{s}$. Le support initial du catalyseur était une silice atomisée de $d_{50} \cong 70 \mu \mathrm{m}$. Une évolution de la teneur en particules fines $(<40 \mu \mathrm{m}) \mathrm{de}$ 7 à $20 \%$, a abaissé $H_{O K}$ de 3,4 à 2 m (fig. 14).

Van Swaaij et Zuiderweg (1972) ont utilisé la décomposition de l'ozone catalysée par de l'oxyde de fer dans des appareils de $0,05,0,10,0,23,0,30$ et $0,60 \mathrm{~m}$ de diamètre. Les supports du catalyseur ont été (fig. 7) un sable de $d_{50}=210 \mu \mathrm{m}$ et une silice atomisée de $d_{50}=70 \mu \mathrm{m}$. Dans leurs expériences, $h$ a évolué de 0,3 à $3 \mathrm{~m}(1,4 \mathrm{~m}$ pour le sable), $U$ de 0,03 à $0,20 \mathrm{~m} / \mathrm{s}, 1 / \mathrm{k}_{\mathrm{o}}$ de 2 à $0,1 \mathrm{~s}$.

- Avec le sable $\left(d_{50}=210 \mu \mathrm{m}\right)$, dans $D_{T}=0,23 \mathrm{~m}$, à $U=0,15 \mathrm{~m} / \mathrm{s}$ et $h<1,4 \mathrm{~m}, H_{K}$ s'est montré indépendant de la teneur (de 3 à 16\%) en particules fines de moins de $40 \mu \mathrm{m}$ de diamètre.

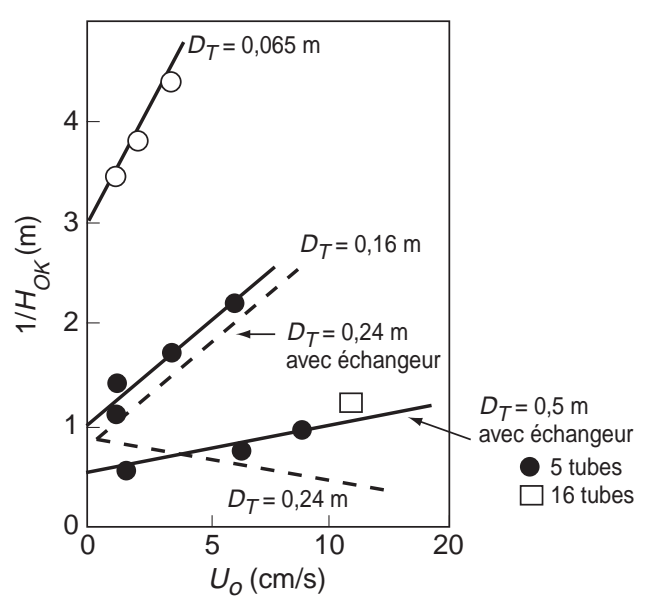

Figure 13

Effet sur $H_{O K}$ (chimie) de la dimension des particules du catalyseur (repérée par $U_{o}$ mesuré à $20^{\circ} \mathrm{C}$ ), du diamètre du réacteur et de l'immersion d'un échangeur selon Botton (1970).

Variation of $H_{O K}$ (Chemistry) against the particles diameter of catalyst (plotted by $U_{o}$ measured at $20^{\circ} \mathrm{C}$ ), the reactor diameter and the presence or not of heat transfer tubes. (Botton 1970). 


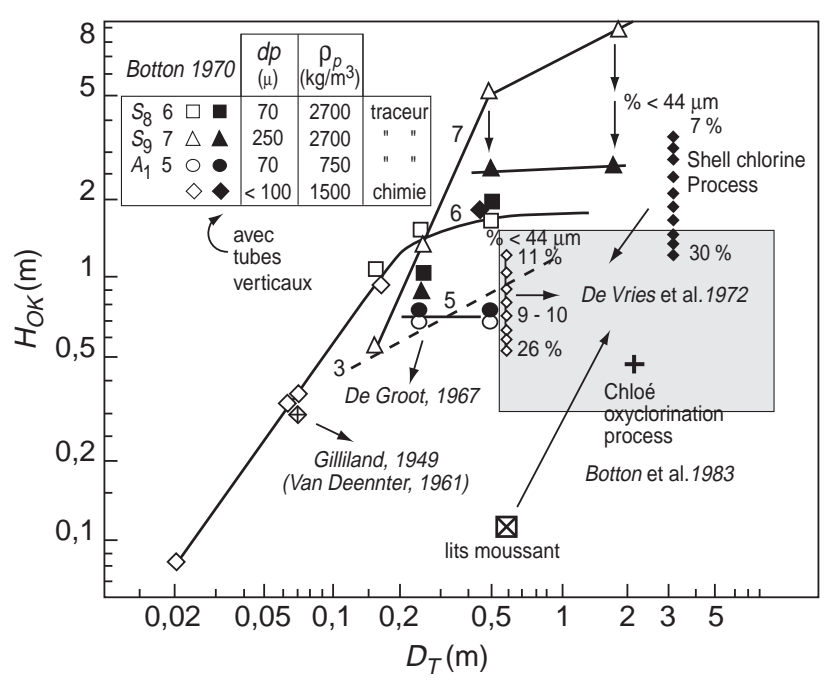

Figure 14

Hauteur de l'unité de transfert $H_{O K}$ dans un lit fluidisé en fonction du diamètre de l'appareil, de la présence ou non de tubes verticaux et de la granulométrie de la poudre. Les numéros de repères sont identiques à ceux de la figure 7 et 11 (granulométries et auteurs). La zone des lits industriels à hautes performances a été ombrée. Ce graphique résume les travaux de Gilliland (1949) interprétés par Van Deemter (1961), de De Groot (1967), de Botton (1970), de De Vries et al. (1972) et de Botton et al. (1983).

Height of unit transfer $H_{O K}$ versus bed diameter, presence (or not) of vertical tubes and powder fluidized. The reference $n^{\circ}$ (granulometries and authors) are the same that on Figures 7 and 11. Area of very high performance industrial beds is shadowed. This figure summarize the works of Gilliland (1949) rewieved by Van Deemter(1961), of De Groot (1967), of Botton (1970), of de Vries et al. (1972) and of Botton et al. (1983).

Les résultats pour $U=0,15 \mathrm{~m} / \mathrm{s}, h<1,5 \mathrm{~m}, 1 / k_{o} \geq 1 \mathrm{~s}$ ont donné la relation :

$$
H_{K}=1,5 h^{0,5} D_{T}^{0,42} \quad(\text { en } \mathrm{m})
$$

- Avec la silice atomisée $\left(d_{50}=70 \mu \mathrm{m}\right)$, dans $D_{T}=0,05$ et $0,23 \mathrm{~m}$ avec $h<1 \mathrm{~m}, H_{K}$ s'est montré proportionnel à $h$ et peu sensible à $D_{T}\left(N_{K}\right.$ toujours $\cong 3$ pour $\left.h \cong 1 \mathrm{~m}\right)$. Dans $D_{T}=0,3$ et $0,6 \mathrm{~m}$ l'influence de $h$ est plus faible $(1<h<3 \mathrm{~m})$ mais celle de $D_{T}$ apparaît $\left(H_{K}=0,6\right.$ et 0,9 dans $D_{T}=0,3$ et $0,6 \mathrm{~m}$ pour $h=2 \mathrm{~m}$ ).

- Lorsque $1 / k_{o}$ diminue, $H_{K}$ reste constant pour $1 / k_{o} \geq 0,5 \mathrm{~s}$ avec la silice atomisée $\left(d_{50}=70 \mu \mathrm{m}\right)$ et $1 / k_{o} \geq 1 \mathrm{~s}$ avec le sable $\left(d_{50}=210 \mu \mathrm{m}\right)$ ensuite il diminue. Une diminution de $20 \%$ de $H_{K}$ demande une diminution de $1 / k_{o}$ de 0,5 à $0,125 \mathrm{~s}$ avec la silice atomisée et de 1 à $0,5 \mathrm{~s}$ avec le sable. Les mesures ont été faites avec $D_{T}=0,23 \mathrm{~m}$ à $0,10<U<0,15 \mathrm{~m}$ et $h \cong 1 \mathrm{~m}$.

\subsubsection{Mesures de $K_{o}\left(H_{O K}\right)$ et $E_{o}\left(H_{O E}\right)$ avec des traceurs}

Gilliland et Mason (1952) ont utilisé des injections de type échelon et permanent sur un lit de $0,075 \mathrm{~m}$ de diamètre. Leurs résultats ont été interprétés par Van Deemter (1961). Nous avons retenu ceux qui concernent les fractions de 70 et $102 \mu \mathrm{m}$ d'un catalyseur de craquage catalytique.

Les valeurs de $N_{E} / N_{K} \cong H_{O K} / H_{O E}$ trouvées par Van Deemter sont, aux vitesses de gaz de $0,12,0,18$ et $0,27 \mathrm{~m} / \mathrm{s}$ de 4, 5 et 7-9 pour chacune des deux poudres. Celles de $H_{K}$, exprimées en hauteur de lit, conduisent, après transformation en hauteur de phase dense (faite en supposant que le lit pistonnait), à une valeur unique pour les deux poudres et les trois vitesses de gaz. Cette valeur est $H_{O K}=0,32 \mathrm{~m}$.

De Groot (1967) a expérimenté avec deux poudres de silice broyées de répartitions granulométriques différentes et de $d_{50}$ voisin (150 et $200 \mu \mathrm{m}$, fig. 7), dans des maquettes de $0,1,0,3,0,6$ et $1,5 \mathrm{~m}$ de diamètre, à des vitesses de gaz de 0,1 et $0,2 \mathrm{~m} / \mathrm{s}$.

Il a utilisé l'écriture de Van Deemter. Le coefficient de dispersion $E$ a été mesuré avec un solide radioactif (méthode de May) et $K$, connaissant $E$, avec un traceur gaz en utilisant la méthode impulsion de Van Deemter. Pour exploiter le signal de sortie un agitateur pour homogénéiser les gaz au-dessus du lit a été nécessaire.

- Pour la poudre à granulométrie étalée :

Les valeurs de $H_{K}$ obtenues pour $0,1<D_{T}<1,5 \mathrm{~m}$, $1<h<5 \mathrm{~m}$ et $0,1<U<0,2 \mathrm{~m} / \mathrm{s}$ satisfont la relation :

$$
H_{K}=0,67 D_{T}^{0,25} h^{0,50} \quad \text { (en m) }
$$

Les valeurs de $N_{E} / N_{K}$ ont évolué pour $U=0,2 \mathrm{~m} / \mathrm{s}$ de 4,5 (avec $\left.D_{T}=0,1 \mathrm{~m}\right)$ à $2,10\left(\operatorname{avec} D_{T}=1,5 \mathrm{~m}\right)$. À $U=0,1 \mathrm{~m} / \mathrm{s}$ elles sont plus faibles.

- Pour la poudre de granulométrie peu dispersée, les valeurs de $N_{E} / N_{K}$ sont beaucoup plus grandes. Celles de $H_{K}$ sont plus faibles pour $D_{T}<0,3 \mathrm{~m}$ et plus élevées pour $D_{T}>0,3 \mathrm{~m}$.

De Vries et al. (1972) ont utilisé les mêmes techniques que De Groot (1967). La silice broyée proposée par De Groot ayant dû être abandonnée pour préparer le catalyseur du Shell Chlorine Process, ils ont pris une silice atomisée $\left(d_{50} \cong 70 \mu \mathrm{m}\right)$ avec laquelle ils ont étudié l'incidence de la teneur en particules fines $(<44 \mu \mathrm{m})$, de la température, de la pression et de la nature du gaz traceur.

- Avec $D_{T}=0,6 \mathrm{~m}$ à $U=0,15 \mathrm{~m} / \mathrm{s}$ et avec $h=3 \mathrm{~m}$, une augmentation de la teneur en particules fines $(<44 \mu \mathrm{m}) \mathrm{de}$ $11 \%$ et $22 \%$ a diminué $H_{O K}$ de 1,25 à $0,52 \mathrm{~m}\left(N_{E} / N_{K}\right.$ de $4,5$ à 1,1$)$ à $20^{\circ} \mathrm{C}$ et de 1 à $0,30 \mathrm{~m}\left(N_{E} / N_{K}\right.$ de 2,2 à 0,5$)$ à $360^{\circ} \mathrm{C}$.

- La même valeur de $H_{O K}$ a été obtenue avec deux gaz traceurs (argon et hélium) de coefficients de diffusion différents (rapport 1/4) avec $D_{T}=0,1 \mathrm{~m}, h=2,2 \mathrm{~m}, 15 \%$ de particules $<44 \mu \mathrm{m}$ et $U=0,1 \mathrm{~m} / \mathrm{s}$. 
- L'expansion du lit diminue lorsque la pression s'abaisse en dessous de 0,8 bar absolu $\left(D_{T}=0,1 \mathrm{~m}\right.$, $\cong 20 \%$ de particules $<44 \mu \mathrm{m}, U=0,18 \mathrm{~m} / \mathrm{s}$ et $P<1,5$ bar).

- Une structure moussante (très petites bulles) a été obtenue à $300^{\circ} \mathrm{C}$ avec une poudre fine, riche en particules inférieures à $20 \mu \mathrm{m}$, obtenue par attrition de la silice atomisée étudiée (voir $\mathrm{n}^{\circ} 11$, fig. 7).

Il est possible, selon De Vries, que les forces d'agglomérations des particules (qui seraient dans ce cas précis, selon De Vries, la conséquence de l'adsorption de gaz à $20^{\circ} \mathrm{C}$ ) disparaissent à $300^{\circ} \mathrm{C}$. Dans cet état, la fluidisation est douce et les entraînements faibles. Sa stabilité s'est montrée trop sensible à la nature de l'environnement physicochimique des particules pour pouvoir l'utiliser. Pour avoir un point de repère vis-à-vis de cet état, nous lui attribuerons comme borne supérieure pour $H_{K}$, le résultat obtenu à $360{ }^{\circ} \mathrm{C}$ sur le catalyseur fluidisé à l'air : $\left(D_{T}=0,6 \mathrm{~m}, U=0,11 \mathrm{~m} / \mathrm{s}, 26,2 \%\right.$ $<44 \mu \mathrm{m}, h_{o} / h_{m f}=1,02, h_{m f}=1,97 \mathrm{~m}, h=2,56 \mathrm{~m}, U_{B X}=0,42$ $\left.\mathrm{m} / \mathrm{s}, H_{O K}=0,11 \mathrm{~m}, N_{E} / N_{K}=0,16\right)$. L'expansion de la phase dense ne se distingue pas ici de celle du lit.

Van Swaaij et Zuiderweg (1972) puis Bohle et Van Swaaij (1978) ont utilisé les mêmes méthodes que De Groot (1967) et De Vries et al. (1972).

- Les mesures de Van Swaaij et Zuiderweg ont été faites avec du sable $\left(d_{50}=210 \mu \mathrm{m}\right)$. Les valeurs de $H_{K}$ trouvées sont voisines de celles obtenues en utilisant la décomposition catalytique de l'ozone (voir ci-dessus). Celles de $N_{E} / N_{K}$ ont été de 4,1 et 6,2 pour $U=0,09$ et $0,17 \mathrm{~m} / \mathrm{s}, 4 \%<44 \mu \mathrm{m}$ et $D_{T}=0,6 \mathrm{~m}$.

- Selon eux, leurs résultats (silice atomisée), ceux de De Vries (silice atomisée), et ceux de De Groot (sable à granulométrie étalée) obtenus avec un gaz traceur ou une réaction chimique sont conformes à la relation :

$$
H_{K}=\left[1,8-\left(1,06 / D_{T}^{0,25}\right)\right]\left[3,5-\left(2,5 / h^{0,25}\right)\right]
$$

Cette relation n'est pas une corrélation, mais un simple repère. $H_{K}$ est susceptible de varier avec la vitesse des gaz, la teneur en particules fines, la pression, la température, le milieu chimique, etc.

- Bohle et Van Swaaij (1978) ont montré que $K$ et $E$ sont indépendant du coefficient de diffusion du gaz traceur et que $K$ augmente si le gaz traceur est adsorbable. Ce dernier point est à rapprocher de la diminution de $H_{K}$ notée cidessus pour les réactions très rapides.

Botton (1970) a utilisé (fig.7), deux sables broyés de $d_{50}=70$ et $250 \mu \mathrm{m}$ avec $d_{10} / d_{50}=1,6\left(S_{8}\right.$ et $\left.S_{9}\right)$ et un support de catalyseur de craquage catalytique $A_{1}$ de $d_{50}=70 \mu \mathrm{m}$. La différence majeure entre $S_{8}$ et $A_{1}$ est la densité des particules $(0,75$ et 2,5$)$. Les diamètres des appareils étaient de 0,16 , $0,25,0,5$, et $1,8 \mathrm{~m}$ de diamètre. La vitesse des gaz a évolué de 0,18 à $0,45 \mathrm{~m} / \mathrm{s}$ et la hauteur des lits de 1,5 à $4,5 \mathrm{~m}$. La méthode utilisée est celle de May (injection échelon et constante de temps $S$ ). L'utilisation d'un traceur solide a été évitée en effectuant les mesures sur deux lits de hauteur différente, toutes les autres conditions étant identiques.

- Les résultats sont portés sur les figures 14, 15 et 16.

- $H_{O K}$ s'est toujours montré indépendant de $\left(U-U_{o}\right)$ $\left(0,18<U-U_{o}<0,45 \mathrm{~m} / \mathrm{s}\right)$.

- Dans les appareils de faible diamètre, $H_{O K}$ est plus petit pour les grosses particules que pour les particules fines. Dans les appareils de grand diamètre, on note l'inverse. Les points d'inversion relatifs à $S_{9}$ et $S_{8}$ se situent vers $D_{T}=0,25 \mathrm{~m}$.

- Pour une poudre donnée, $H_{O K}$ augmente avec $D_{T}$ puis en devient indépendant. Sur la figure 14, on peut lire : avec $A_{1}$ : $H_{O K}=0,75 \mathrm{~m}$ pour $D_{T}>0,25 \mathrm{~m} ;$ avec $S_{8}: H_{O K}=1,5 \mathrm{~m}$ pour $D_{T}>0,5 \mathrm{~m} ;$ avec $S_{9}: H_{O K} \cong 8 \mathrm{~m}$ pour $D_{T}>2 \mathrm{~m}$.

- Si l'on immerge des tubes verticaux pour simuler un échangeur industriel $(\phi=65 \mathrm{~mm}$ disposés en mailles carrées de $2 \phi), H_{O K}$ est, avec $D_{T}=0,5 \mathrm{~m}$, inchangé pour $S_{8}$ et $A_{1}(1,5$ et $0,75 \mathrm{~m})$ mais abaissé de 5 à $3 \mathrm{~m}$ pour $S_{9}$ (et de 8 à $3 \mathrm{~m}$ avec $D_{T}=1,8 \mathrm{~m}$ ).

- Les valeurs de $N_{O E} / N_{O K}$ ont été, pour A1 à $\left(U-U_{o}\right)=$ $0,20 \mathrm{~m} / \mathrm{s}$ de 2,1 avec $D_{T}=0,25 \mathrm{~m}$ et de 1,6 avec $D_{T}=0,5 \mathrm{~m}$ sans tubes verticaux et de 3 avec des tubes. Elles ont été un peu plus élevées pour $S_{8}$ (2,2 au lieu de 1,6 dans $\left.D_{T}=0,5 \mathrm{~m}\right)$ et beaucoup plus pour $S_{9}(\cong 8$ avec $D_{T}=0,16,0,25$ et $\left.1,8 \mathrm{~m}\right)$. Leur tendance est d'augmenter $\operatorname{avec}\left(U-U_{o}\right)$.

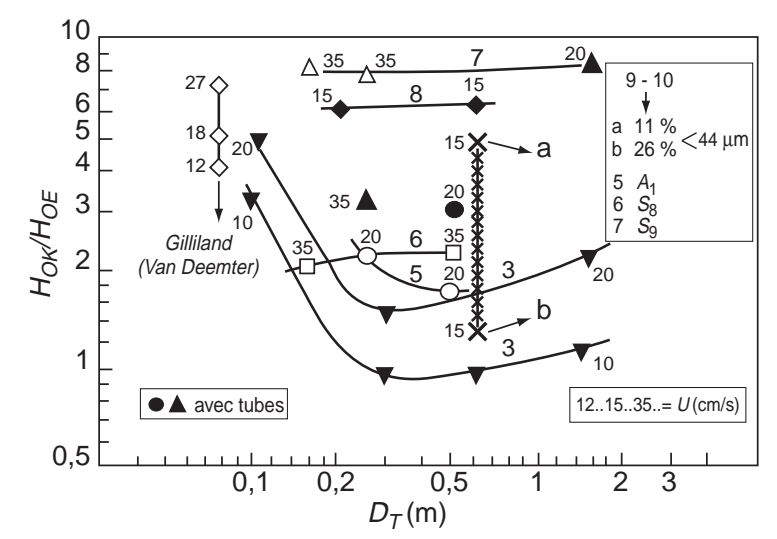

Figure 15

$H_{O K} / H_{O E}$ en fonction de $D_{T},\left(U-U_{o}\right)$ et la présence de tubes verticaux dans les conditions des figures 14 et 11 . Les numéros de repère (granulométries et auteurs) sont ceux de la figure 7. On peut noter que $H_{O K} / H_{O E}$ augmente lorsqu'on immerge des tubes verticaux ou augmente la vitesse des gaz.

$H_{O K} / H_{O E}$ versus $D_{T}\left(U-U_{o}\right)$ and presence of vertical tubes. The conditions are ones of Figures 14 and 11 and reference numbers (granulometries and authors) are ones of Figure 7. It can be seen that $H_{O K} / H_{O E}$ increases when vertical tubes are immersed and when superficial gas velocity is increased. 


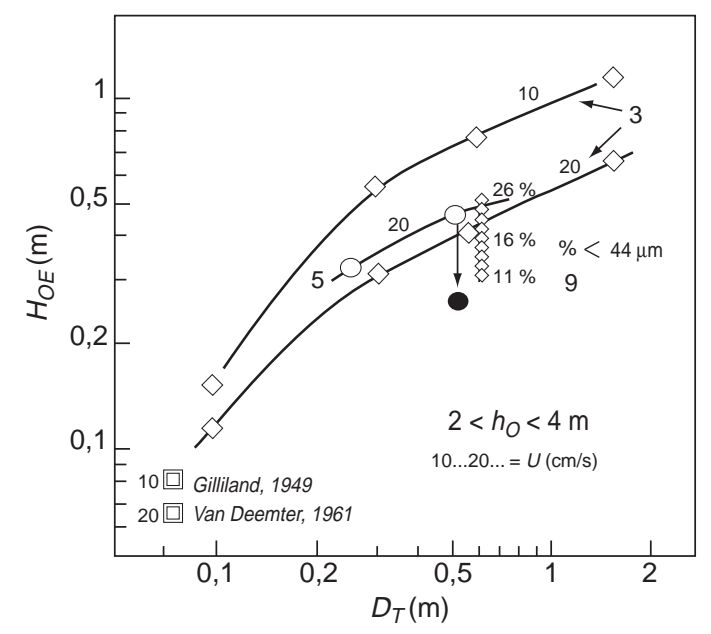

Figure 16

Valeurs de $H_{O E}$ en fonction de $D_{T}$, et de $\left(U-U_{o}\right)$. Effet de la présence de tubes verticaux (point noir) pour des poudres fines. Les numéros sont ceux des figures 7, 14 et 15.

Variations of $H_{O E}$ against $D_{T},\left(U-U_{o}\right)$, and presence of vertical tubes for fine particles. The reference numbers (granulometries and authors) are ones of Figures 7, 14 and 15.

\subsubsection{Dispersion axiale et hauteur d'unité de mélange : commentaires}

Les premières mesures de $D_{S}$ ont été faites par May (1959) avec un traceur solide sur des lits de particules fines fluidisés à $U=0,20 \mathrm{~m} / \mathrm{s}$. Les diamètres des lits étaient de $0,075,0,38$ et $1,5 \mathrm{~m}$ et leur hauteur de $10 \mathrm{~m}$. Il a trouvé respectivement $0,055,0,15$ et $0,46 \mathrm{~m}^{2} / \mathrm{s}$. Ces valeurs signifient selon May que 50 grammes de catalyseur se mélangent en moins d'une minute à 15 tonnes de catalyseur dans un appareil de $1,5 \mathrm{~m}$ de diamètre.

Sur la figure 16, quelques valeurs de $H_{O E}$ ont été portées en fonction de $D_{T}$ à $U=0,10$ et $0,20 \mathrm{~m} / \mathrm{s}$. Elles sont relatives à des poudres donnant une fluidisation de qualité. On notera une bonne cohérence entre les auteurs. $H_{O E}$ diminue lorsque le diamètre de l'appareil diminue, la vitesse des gaz augmente et que l'on immerge un échangeur.

\section{INTERPRÉTATION - TENDANCES À SUIVRE - EXTRAPOLATION}

Les résultats décrits ci-dessus ont été traduits en cinq graphiques. Ils donnent les granulométries des poudres étudiées (fig. 7) et, pour ces poudres, en fonction de $D_{T}: U_{B X}$ (fig. 10), $H_{O K}$ (fig. 14), $N_{O E} / N_{O K}$, (fig. 15) et $H_{O E}$ (fig. 16). Ils constituent un guide dont on peut extraire des ordres de grandeur, des tendances à suivre et évaluer les performances possibles. Ils sont centrés sur les poudres à particules fines. Ce sont les seules qui, pour les lits catalytiques à hautes performances sont utilisables. La synthèse qui suit en facilitera l'utilisation.

- Les valeurs de $H_{O K}$ ont un effet décisif sur l'avancement $R$ d'une réaction. Celui de $N_{O E} / N_{O K}$ est négligeable mais il peut ne pas l'être sur la chimie des systèmes de réactions complexes.

- Les valeurs de $N_{O E} / N_{O K}$ ont tendance à augmenter avec $\left(U-U_{o}\right)$. Celles de $U_{B X}$ et de $H_{O K}$ en sont indépendantes pour $\left(U-U_{o}\right)>0,12\left(g D_{T}\right)^{0,5}$ dans les lits de laboratoire et $\left(U-U_{o}\right)>0,10 \mathrm{~m} / \mathrm{s}$ dans les lits de $D_{T}>0,5 \mathrm{~m}$.

- Les valeurs de $U_{B X}, H_{O K}, N_{O E} / N_{O K}$, et $H_{O E}$ évoluent lorsque $D_{T}$ augmente puis en deviennent indépendantes. Le diamètre de transition s'abaisse lorsque la qualité de la fluidisation s'améliore. Il est plus élevé pour $N_{O E} / N_{O K}$ et $H_{O E}$ que pour $U_{B X}$ et $H_{O K}$. Donnons deux exemples de diamètre de transition pour $H_{O K}$ déduits de la figure 14 :

$$
\begin{aligned}
& D_{T} \cong 0,25 \mathrm{~m} \text { pour } H_{O K}=0,75 \mathrm{~m} \\
& \text { (poudre } A_{1}, d_{50}=70 \mu \mathrm{m}, d_{10} / d_{50}=1,6, d a_{p}=0,75 \text { ) } \\
& D_{T} \cong 0,50 \mathrm{~m} \text { pour } H_{O K}=1,5 \mathrm{~m} \\
& \text { (poudre } S_{8}, d_{50}=70 \mu \mathrm{m}, d_{10} / d_{50}=1,6, d a_{p}=2,50 \text { ) }
\end{aligned}
$$

- Les valeurs de $H_{O K}$ mesurées avec des traceurs autorisent l'obtention de très hauts taux de conversion dans les réacteurs industriels. Mais il faut savoir réaliser pour un procédé donné des valeurs de $H_{O K}>0,3$ m pour être certain d'éviter les lits moussant et $<1 \mathrm{~m}$ pour s'assurer, avec des couches fluidisées de 10 à $15 \mathrm{~m}$, de disposer de $N_{O K}>8$ ( $R>99,9$ accessible).

- La réalisation de $0,3<H_{O K}<1 \mathrm{~m}$ implique l'utilisation de poudres fines pour lesquelles les forces de cohésion entre les particules (fonctions de leur environnement physicochimique) ont un effet non négligeable sur la qualité de la fluidisation (donc sur $H_{O K}$ ). Les mises au point à faire sont, de ce fait, spécifiques au procédé considéré.

- Les facteurs d'action pour diminuer $H_{O K}$ sont : la finesse moyenne du catalyseur, la teneur en particules fines (en catalyseur, support de catalyseur ou autres poudres), la densité apparente, la porosité et sa morphologie. (La répartition de la matière active du catalyseur sur le support est très importante si elle est fluide).

- $H_{O K}$ est indépendant de la pression (de 1 à 5 bar), du coefficient de diffusion des réactifs et de la constante cinétique de la réaction chimique (pour $1 / k_{o} \geq 0,25 \mathrm{~s}$ ).

- $H_{O K}$ n'est pas modifié dans un grand appareil si on immerge un échangeur de type industriel $(\phi=89 \mathrm{~mm}$, maille carrée de $2 \phi, D_{T}=0,5 \mathrm{~m}$ ) mais peut l'être (diminué) dans des appareils plus petits avec des échangeurs plus compacts $\left(\phi=17 \mathrm{~mm}\right.$, maille carrée de $\left.2 \phi, D_{T}=0,25\right)$. La notion de diamètre hydraulique est inapplicable. $N_{O E} / N_{O K}$ augmente dans les deux cas.

- En laboratoire $\left(D_{T} \leq 0,10 \mathrm{~m}\right) H_{O K}$ ne dépend pas de la granulométrie (si $d_{50}<150 \mu \mathrm{m}$ ). On observe l'inverse dans les pilotes $\left(D_{T} \geq 0,25 \mathrm{~m}\right)$. 
- Les propriétés obtenues incitent à travailler à haute vitesse de gaz. $N_{O K}$ reste constant et $N_{O E} N_{O K}$ augmente lorsque la vitesse des gaz augmente. En conséquence, l'effet du mélange du gaz de la phase dense diminue. Ce dernier point est intéressant si la réaction est complexe.

Si les tendances dégagées ci-dessus ont été suivies, les résultats obtenus dans un pilote de $0,5 \mathrm{~m}$ de diamètre se conservent si l'on augmente le diamètre, toute autre condition étant inchangée (hauteur du lit, etc.). Des performances très élevées sont accessibles si l'on arrive à maîtriser le transfert entre la phase bulles et le gaz de la phase dense en faisant évoluer les propriétés physiques du catalyseur.

Il est impératif qu'en toute circonstance, le distributeur de gaz et les cyclones remplissent bien leurs missions. Ceci est très important si le diamètre de l'installation est grand et si les perormances du catalyseur en contact gaz-solide sont limites. Un médiocre distributeur amplifiera ses faiblesses. À l'inverse, un catalyseur très performant aura tendance à effacer ses défauts.

Les procédés de Shell et de Péchiney - Saint-Gobain ont été positionnés (en valeur de $H_{O K}$ ) sur la figure 14. Les résultats du premier ont été publiés dès la mise en exploitation (De Vries et al. (1972) et ceux du deuxième quelques années après (Botton et al., 1983).

Pour terminer ce sujet d'extrapolation avec un pilote, citons les publications de Massimila et Donsi (1976) et Myauchi et al. (1981) relatives aux phénomènes de base, celle de Bolthrunis (1989) sur la perspective future des modèles, celle de Bergougnou et Backer (1973) sur le calcul des grands lits fluidisés, celle de Holves et al. (1973) de Badger Compagny Inc. sur les applications du lit fluidisé aux réactions chimiques catalytiques. Citons également les indispensables ouvrages de Kunii et Levenspiel $(1969,1993)$ sur la fluidisation en général, et la publication de Botton et Delcoigne (1985) sur la réalisation d'un circuit fermé de plusieurs lits fluidisés en série, avec possibilité de faire circuler à l'intérieur des lits le gaz et les particules à cocourant ou à contre-courant à l'aide de plateaux.

\section{REMERCIEMENTS}

L'auteur remercie M. Pierre Trambouze d'avoir relu le manuscrit et de lui avoir permis, par ses nombreuses suggestions, de beaucoup l'améliorer.

\section{RÉFÉRENCES}

Avidan, A.A. et Yerushalmi, J. (1982) Bed Expansion in High Velocity Fluidization. Powder Technol., 32, 223-231.

Bergougnou, M.A. et Baker, C.G.J. 1973) Étude sur l'extrapolation et le calcul des grands lits fluidisés. Chimie et Industrie - Génie chimique, 106, 4.

Bohle, W. et Van Swaaij, W.P.M. (1978) The Influence of Gas Absorption on Mass Transfert and Gas Mixing in a Fluidized Bed. Fluidization (J.F. Davidson and D.L.Kearns, eds.), 167-172, Cambridge University Press, London and New York.
Bolthrunis, C.O. (1989) An Industrial Perspective on Fluidized Bed Model Reactor. Chem. Eng. Prog., 51-54.

Botton, R. (1970) Gaz Solid Contacting in Fluidized Beds. A.I.Ch.E, Symp Series, 101, 66, 8-18.

Botton, R., Cosserat, D. and Charpentier, J.C. (1978) Influence of Column Diameter and High Gas Throughouts on the Operation of a Bubble Column. Chem. Eng. J., 16, 107-115.

Botton, R. (1980) Réacteurs à lit fluidisé, gaz-solide à haut taux de conversion, miniaturisation de l'échelle pilote. Contrat DGRST (France) Annexes du rapport $\mathrm{n}^{\circ}$ 77-7-1614 (82 pages).

Botton, R., Cosserat, D. et Charpentier, J.C. (1980b) Mass Transfer in Bubble Columns Operating at High Gas Throughputs. Chem. Eng. J., 20, 87-94.

Botton, R. et Vergnes, F. (1981) Catalytic Fluidized Reactor Scale-Up. An Approach from Chemical and Specifically Physical Laboratory Data without the Need of a Pilot Plant Stage. Chem. Ing. Techn., 11, 481 and VDI Berichte, 409, 567-587.

Botton, R. et Delcoigne, A. (1985) Quelques dispositifs utiles pour la réalisation d'un circuit continu fermé de plusieurs lits fluidisés en série. Journées européennes de fluidisation, Toulouse, France.

Botton, R., Benadda, B. et Otterbein, M. (1997) Treatment of Gases and Dust Using Droplet Columns. The Canadian J. of Chem. Eng., 75, 527-534.

Carlsmith, L.E. et Johnson, F.B. (1945). Pilot Plant Development of Fluid Catalytic Cracking. Ind. Eng. Chem., 37, 5, 451-455.

Danckwerts, J.W., Jenkins, J.W. et Place, G. (1954) The Distrib. of Residence Times in a Indust. Fluid. Reactor. Chem. Eng. Sci. 3, 26-35.

Davidson, J.F. et Harrison, D. (1963) Fluidized Particles. Cambridge University Press, Cambridge (UK).

De Groot, J.H. (1967) Scaling-Up of Gas-Fluidized Bed Reactors. Procedding of Inter. Symp. on Fluidization, 348, Eindhoven, Netherland University Press, Amsterdam.

De Vries, R.J., Van Swaaij, W.P.M., Mantoni, C. et Heijkoop, A. (1972) Design Criteria and Performence of the Commercial Reactor for the Shell Chlorine Process. Proceeding of the 5th European Sympos. on Chemical Reaction Engineering, Amsterdam, B9, 59-69. Elsevier Publication.

Gehdhart, D. (1973) Powder Technology, 7, 285-292.

Gilliland, E.R., Mason, E.A. et Oliver, R.C. (1949) Gas and Solid Mixing in Fluidized Bed. Ind. Eng. Chem., 41, 6, 1191-1196.

Gilliland, E.R., Mason, E.A. et Oliver, R.C., (1952) Gas Mixing. In: Beds of Fluidized Solids, 44, 1, 218-224.

Gilliland E.R., Mason, E.A. et Oliver R.C. (1953) Gas-Flow Patterns. In: Beds of Fluidized Solids, 45, 6, 1177-1185.

Grekel, H. et Hujsak, H.L. (1964) Chem. Eng. Prog., Mungen R., 60, 1,56 .

Handlos, A.E., Kunstman, R.W. et Schissler, D.O. (1957) Gas Mixing Characteristics of a Fluid Bed Regenerator. Ind. Eng. Chem. 49, 1, 25-30.

Holve, Sheely et Schaffer (1973) (Badger Compagny Inc.) Application du lit fluidisé aux réactions chimiques. Chimie Actualité, 41-44.

Kunii, D., Levenspiel O. (1968) Bubbling Bed Model. Ind. Eng. Fund., 7, 6, 441-451.

Kunii, D. et Levenspiel, O. (1969) Fluidization Engineering, Wiley New York.

Kunii, D. et Levenspiel, O. (1993) Fluidization Engineering, Butterworth-Heinmann, Boston, MA.

Levenspiel, O. (1962) Chemical Reaction Engineering, Wiley New York. 
Lewis, W.K., Gilliland, E.R. et Werner Glass (1959) SolidCatalyzed Reaction in a Fluidized Bed. AIChE Journal, 5, 4, 419-426.

Massimila, L. et Donsi, G. (1976) Cohesive Forces beetween Particles of Fluid Bed Catalyst. Powder Technology, 15, 253-260.

Matheson, G.L., Herbst, W.A. et Holt, P.H. (1949) Caracteristics of Fluid and Solid Systems. Ind. Eng Chem., 41, 6, 1089-1100.

Mathis, J.F. et Watson, C.C. (1956) AIChE Journal, 2, 518.

Matsen, J.M. (1970) Expansion of Fluidized Beds in Slug Flow. AIChE Symp. Series, 101, 66, 47-51.

Matsen, J.M. (1973) Evidence of Maximum Bubble Size in a Fluidized Bed. AIChE. Symp. Series n ${ }^{\circ} 126,69,30-33$.

May, W.G. (1959) Fluidized Bed Reactors Studies. Chem. Eng. Prog., 55, 12, 49-55.

Miyauchi, T., Furusaki, S., Morooka, S. et Ikeda, Y. (1981) Transport Phenomena and Reaction in Fluidized Catalytic Beds. In: Advances in Chem. Eng., 11, Academic Press New York.

Murphree, M.E.V. (1949) La fluidisation et ses applications. Conférence à l'Association française des techniciens du pétrole, Paris.

Patridge, B.A. et Rowe, P.N. (1966) Transt. Inst. Chem. Eng., 44, 335
Reh, L. (1971) Chem. Eng. Prog., 67, 258.

Sergiou, L. et Laguerie, C. (1983) Proc. 4th Inter. Conf. of Fluidization (Engineering Foundation), Kashikojima, Japan, 557-564.

Shen, C.Y. et Johnstone, H.F. (1955) AIChE Journal, 1, 349.

Singer, E., Todd, D.B. et Guinn, V.P. (1957) Catalyst Mixing in Commercial Catalytic Cracking Units. Ind. Eng. Chem., 49, 1, 11-19.

Van Deemter, J.J. (1961) Mixing and Contacting in Gas-Solid Fluidized Beds. Chem. Eng. Sci., 13, 143-154.

Van Swaaij, W.P.M. et Zuiderweg, F.J. (1972) Investigation of Ozone Decomposition in Fluidized Bed on the Basis of the TwoPhase Model. Proceeding of the 5th European Sympos. on Chemical Reaction Engineering, B9, 25-35. Amsterdam, Elsevier Publication Co.

Werther, J. (1980) Modeling and Scale-Up of Industrial Fluidized Bed Reactors. Chem. Eng. Sci., 35, 372-379.

Yerushalmi, J., Turner, D.H. et Squires, A. (1976) The Fast Fluidized Bed. Ind. Chem. Process Des. Dev., 15, 1.

Zenz, F.A. et Othmer, D.F. (1960) Fluidization and FluidParticles System, Reinhold Publishing Corp., New York.

Final manuscript received in July 1999 